\title{
Das Anthropozän-Konzept
}

\author{
Kontrakt - Komposition - Konflikt \\ Jens Kersten"
}

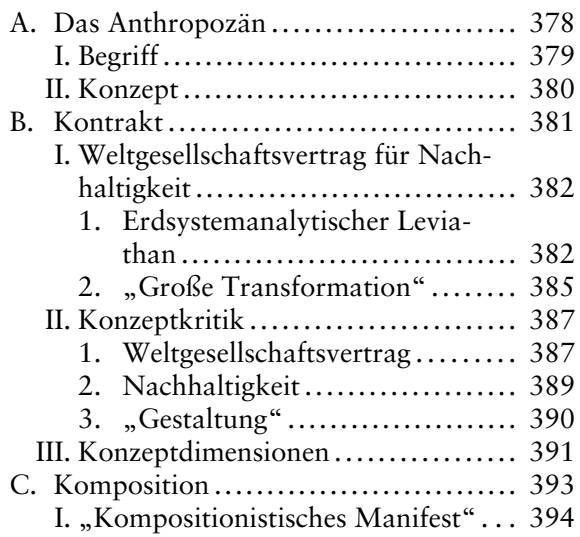

II. Konzeptkritik ..................... 397

1. „Rechtsstaat der Natur“ “....... 397

2. Nichtreflexive Loops............ 400

3. Risikovorsorge ................. 401

III. Konzeptdimensionen ............... 404

D. Konflikt.......................... 405

I. Konfliktkultur ......................... 405

II. Konflikttypen ..................... 406

1. Resistenz ..................... 407

2. Resilienz ..................... 408

3. Persistenz ..................... 409

III. Konfliktakteure .................. 410

1. Menschliche Akteure ........... 410

2. Nichtmenschliche Akteure...... 411

IV. Konzeptdimensionen .............. 412

E. Fazit ................................ 413

\section{A. Das Anthropozän}

Die Erde sei - sagen die Geowissenschaften - in ein neues Zeitalter eingetreten. Auf das Holozän folge nun das „Anthropozän“, in dem sich der Mensch zu einer geologischen Kraft entwickelt habe. Doch was bedeutet die Ausrufung dieser neuen Erdepoche für die politische und rechtliche Governance unseres Planeten? Das Anthropozän-Konzept veranschaulicht den Wandel des globalen Zeit- und Raumverständnisses. Es reflektiert ein neues Verhältnis von Natur und Kultur. Es überholt die Leitideen der „Risikogesellschaft“ und der „Nachhaltigkeit“. Es fragt nach der normativen Steuerungskraft der Resistenz, Resilienz und Persistenz ökologischer und kultureller Systeme. Es rechnet mit Tieren, Pflanzen und Landschaften als neuen Akteuren im Rechtssystem. Die environmental humanities rekonstruieren die anthropozäne Herausforderung als Kontrakt, Komposition oder Konflikt: Doch welchem dieser drei Anthropozän-Konzepte sollten wir in rechtlicher Perspektive folgen?

* Prof. Dr. Jens Kersten lehrt Öffentliches Recht und Verwaltungswissenschaften an der Juristischen Fakultät der Ludwig-Maximilians-Universität München. Der Autor dankt dem Rachel Carson Center for Environment and Society an der Ludwig-Maximilians-Universität München und insbesondere Christof Mauch und Helmuth Trischler. 


\section{Begriff}

Der Nobelpreisträger Paul Crutzen hat den Begriff des „Anthropozän“ in der Wende vom 20. zum 21. Jahrhundert geprägt: ${ }^{1}$ Der Mensch ist zu einer geologischen Kraft geworden. Deshalb gibt er einer neuen Erdzeit seinen Namen: „Anthropozän“. Mit dieser Begriffsbildung sieht sich Crutzen in einer epistemologischen Tradition: ${ }^{2}$ Der italienische Geologe Antonio Stoppani hatte schon 1873 von einer „anthropozooischen Ära“ gesprochen. Ab den 1920er Jahren kam der Begriff der „Noosphäre“ auf, der von dem russischen Geochemiker Vladimir I. Vernadskij ${ }^{3}$ und dem französischen Theologen Pierre Teilhard de Chardin 4 entfaltet wurde: die "Noosphäre“ als die „Welt des Denkens“, die mit der Natur die Zukunft gestaltet. In den 1960er Jahren hat Rachel Carson den anthropozänen Wandel des Verhältnisses der Menschen zu und in ihrer Umwelt beschrieben: „Nur innerhalb des kurzen Augenblicks, den das jetzige Jahrhundert darstellt, hatte eine Spezies - der Mensch - erhebliche Macht erlangt, die Natur ihrer Welt zu verändern. "5 Anfang der 1990er Jahren diagnostizierte der Biologe Hubert Markl einen „Umbruch ins Anthropozoikum “, 6 in dem die „Natur als Kulturaufgabe“ verstanden werden könne. ${ }^{7}$ In diesem begriffsgeschichtlichen Kontext entwickelt Crutzen sein Verständnis des Anthropozän als die „present, in many ways human-dominated, geological epoch, supplementing the Holocene - the warm period of the past 10-12 millennia. " 8 Wesentlich für diesen Ausweis einer neuen Erdepoche ist nicht die schlichte Tatsache, dass Menschen die Erde seit Jahrtausenden (mit)gestalten. Vielmehr sind die Menschen im Anthropozän in ihrer Kultur- und Naturgestaltung selbst zu einer geologischen Kraft geworden. "It is time“ - so Crutzen zusammen mit Jacques Grinevald, John McNeill und Will Steffen - „to recognize the Anthropocene as a new epoch in Earth history due to the global-scale of human influence on the environment. "9 Die Modifikation von biochemischen und Wasserkreisläufen, die unwiederbringliche Vernichtung von Biodiversität, die Veränderung des Weltklimas, die Transformation von Landschaften sowie Genetic Engineering und synthetische Biologie charakterisieren das Anthropozän, das sich nach der Auffassung von Crutzen et al. in drei Schritten entwickelt

1 Vgl. hierzu und zum Folgenden P. J. Crutzen, Geology of mankind: the Anthropocene, Nature 415 (3.1.2002), S. 23; ders./E. F. Stoermer, The „Anthropocene“, Global Change Newsletter 41 (2000), S. $17 \mathrm{f}$.

2 Vgl. hierzu und zum Folgenden W. Steffen/J. Grinevald/P. J.. Crutzen/J. McNeill, The Anthropocene, Philosophical Transactions of the Royal Society 369 (2011), S. 842 (843 ff.); C. Schwägerl, Menschenzeit, München 2012, S. 25 ff.; jew.m.umf.N.

3 V. I. Vernadskij, Der Mensch in der Biosphäre, Frankfurt u.a. 1997, S. 147 ff., 209 ff., 239 ff.

4 P. Teilhard de Chardin, Der Mensch im Kosmos, 5. Aufl., München 1988, S. 193 ff.

5 R. Carson, Der stumme Frühling, München 2012, S. 18.

6 H. Markl, Natur als Kulturaufgabe, Stuttgart 1991, S. 319 ff.

7 Vgl. E. Ehlers, Das Anthropozän, Darmstadt 2008, S. 13.

8 Crutzen, Geology (Fn. 1), S. 23.

9 Steffen/Grinevald/Crutzen/McNeill, Anthropocene (Fn. 2), S. 842. 
hat: ${ }^{10}$ Die erste Periode habe mit der Industrialisierung eingesetzt und datiere deshalb von 1800 bis 1945. Nach dem Zweiten Weltkrieg sei es zu einer Beschleunigung der Entwicklung gekommen und das Anthropozän in eine zweite Phase der „Great Acceleration"11 eingetreten: Die Explosion der Weltbevölkerung, globale Infrastrukturierung und ökonomische Globalisierung sowie wohlfahrtsgesellschaftliche Konsumeskalation gehen Hand in Hand mit dem Kollaps von Ökosystemen, der Verschwendung von Ressourcen, dem Artensterben und der Erderwärmung. Die dritte Phase des Anthropozän beginne schließlich in unseren Tagen als Periode der „growing awareness of human impact on the environment at the global scale and the first attempts to build global governance systems to manage humanity's relationship with the Earth System." 12 Zugleich äußern Crutzen et al. aber mit Blick auf das Scheitern einer nachhaltigen Klima-Governance und der Bewahrung der Artenvielfalt Zweifel an der Konsistenz dieser dritten Anthropozän-Stufe - ein Zweifel, der die Autoren zu ihrer Ausgangsfrage zurückführt: „Can human activity really be significant enough to drive the Earth into a new geological epoch?"13 Und in ihrer Antwort auf diese Frage vergleichen sie Charles Darwins Verständnis der Evolution mit ihrer Perzeption des Anthropozän: „Darwin's insight into our origins provoked outrage, anger and disbelief but did not threaten the material existence of society of the time. The ultimate drivers of the Anthropocene, on the other hand, if they continue unabated through this century, may well threaten the viability of contemporary civilisation and perhaps even the future existence of Homo sapiens. "14

\section{Konzept}

Das Anthropozän unterscheidet sich in einem zentralen Punkt von seinen Vorgängern: Erdzeitalter werden in der Regel retrospektiv bestimmt und in den meisten Fällen nach dem ausgestorbenen Leitfossil benannt. Im Gegensatz dazu bringt das Anthropozän die erdgeschichtliche Gegenwart auf einen Begriff, und die Menschheit ist (bisher) noch nicht ausgestorben. Aus diesen beiden Gründen kommt das Anthropozän nicht ohne eine Form der menschlichen Selbstreflexion aus. Ganz in diesem Sinn beschreiben auch Crutzen, Grinevald, McNeill und Steffen nicht nur die naturwissenschaftliche Faktizität der anthropozänen Entwicklung. Sie sprechen von einem „Anthropocene concept“: 15 Wenn der Mensch zu einem zentralen geologischen Faktor der Erde geworden ist, muss er zugleich eine konzeptionelle Vorstellung davon entwickeln, wie er seiner erdgeschichtlichen „Rolle“ gerecht werden will. Diese Reflexivität des Anthropozän-Konzepts lässt sich in vier Dimensionen entfal-

10 Vgl. Steffen/Grinevald/Crutzen/McNeill, Anthropocene (Fn. 2), S. 847 ff.

11 Steffen/Grinevald/Crutzen/McNeill, Anthropocene (Fn. 2), S. 849 ff.

12 Steffen/Grinevald/Crutzen/McNeill, Anthropocene (Fn. 2), S. 856.

13 Steffen/Grinevald/Crutzen/McNeill, Anthropocene (Fn. 2), S. 862.

14 Steffen/Grinevald/Crutzen/McNeill, Anthropocene (Fn. 2), S. 862.

15 Steffen/Grinevald/Crutzen/McNeill, Anthropocene (Fn. 2), S. 843. 
ten. Erstens ist das Anthropozän in zeitlicher Hinsicht reflexiv: Es ist die vergängliche Erdzeit der Menschen. Zweitens ist das Anthropozän in räumlicher Hinsicht reflexiv: Die Menschen werden sich der räumlichen Endlichkeit der Erde als „ihres“ Planeten bewusst. Drittens ist das Anthropozän im Hinblick auf den Menschen als seinen zentralen Akteur reflexiv: Die Menschen greifen in die ökologischen Arten, Kreisläufe, Ressourcen und Systeme ein und verändern damit zugleich auch ihre eigene Welt. Viertens ist das Anthropozän im Hinblick auf menschliches Wissen reflexiv: Vor allem die Wissenschaft kann versuchen, die (un)vorhersehbaren Folgen menschlichen Handelns im Anthropozän zu bestimmen und mit ihnen konstruktiv umzugehen.

Diese vier Dimensionen anthropozäner Reflexivität - Zeit, Raum, Akteur und Wissen - verdeutlichen, warum sich das Anthropozän im Unterschied zu anderen Erdzeitaltern nicht nur mit einer faktischen Beschreibung begnügen kann, sondern gleichsam automatisch ein reflexives Konzept von ethischen Grundsätzen und rechtlicher Governance erfordert. Ganz in diesem Sinn haben auch Crutzen et al. in ihrer Periodisierung des Anthropozän dessen dritte - bisher enttäuschende - Phase als die Zeit anthropozänen Bewusstseins und anthropozäner Governance ausgewiesen: Die reflexive Realisierung von Handlungsoptionen resultiert in ethischer Verantwortung und rechtlichen Regelungen. Der Versuch, die ethischen und rechtlichen Möglichkeiten sowie Grenzen menschlichen Handelns im Anthropozän konzeptionell auszuloten, fällt nicht leicht, ist aber unumgänglich. So hat Christian Schwägerl gefordert, dass sich einzelne Individuen und ganze Gesellschaften nicht so benehmen sollen, als seien sie die letzten auf diesem Planeten. ${ }^{16}$ Gerade im Hinblick auf das Verhalten der westlichen Wohlstandsgesellschaften ist dies schon viel verlangt. Doch angesichts des changierenden Verhältnisses von Kultur und Natur im Anthropozän ist die Frage nach dem anthropozänen Imperativ noch komplexer: Welche Lebensstile sind in einer Welt globalisierbar, die die tradierte Unterscheidung von Natur und Kultur verloren zu haben scheint? Die Antworten, die gegenwärtig in den environmental humanities auf diese Frage gegeben werden, variieren sehr stark danach, wie die vier soeben genannten Dimensionen anthropozäner Reflexivität konzeptionell gefasst werden. Im Folgenden sind deshalb drei Anthropozän-Konzepte vorzustellen und zu diskutieren: Kontrakt, Komposition und Konflikt.

\section{B. Kontrakt}

Das kontraktualistische Anthropozän-Konzept entwirft einen Weltgesellschaftsvertrag für nachhaltige Entwicklung. 


\section{Weltgesellschaftsvertrag für Nachhaltigkeit}

Hans Joachim Schellnhuber hat den Weg vom „modernen“ zum „post-anthropozänen Leviathan" vorgezeichnet und damit maßgeblich den Vorschlag des Gesellschaftsvertrags für eine Große Transformation geprägt, den der Wissenschaftliche Beirat der Bundesregierung Globale Umweltveränderungen (WBGU) entworfen hat.

\section{Erdsystemanalytischer Leviathan}

In seinem Artikel über „Earth system" analysis and the second Copernican revolution, der im Millenniumsupplement von Nature im Jahr 1999 publiziert wurde, kündigte Hans Joachim Schellnhuber die „emergence of a modern ,Leviathan'“17 an. Schellnhuber formulierte sein Modell zunächst nicht mit einer ausdrücklichen Referenz zum Begriff des „Anthropozän“, dessen Konzept von Paul Crutzen zeitgleich profiliert wurde. Vielmehr spricht Schellnhuber zu diesem Zeitpunkt noch von einem "geo-cybernetic age“ ${ }^{18}$ Doch die anthropozäne Einsicht, dass der human factor zu einem weltweiten „mega-factor" ${ }^{19}$ geworden ist, wird auch von Schellnhuber geteilt und dementsprechend in sein Modell einer Erdsystemanalyse integriert. Vor diesem Hintergrund ist es auch nur konsequent, dass Schellnhuber in den folgenden Jahren den Begriff des „Anthropozän“ argumentativ aufgegriffen ${ }^{20}$ und sein Konzept des „modernen“ zum „post-Anthropocene Leviathan“21 weiterentwickelt hat.

Die faszinierende Grundthese im Theoriedesign Schellnhubers liegt darin, dass der Aufweis des "globalen Subjekts“ - des "modernen Leviathan“ - in die Analyse des Erdsystems integriert wird. ${ }^{22}$ Dieser „moderne Leviathan“ erhebt sich also nicht mehr wie sein Hobbesscher Vorgänger von 1651 als Symbol der Einheit der Staatsgewalt über einer leeren Landschaft, ${ }^{23}$ sondern agiert als ein globales Subjekt nachhaltig im Erdsystem. In der argumentativen Entfaltung der Eckpfeiler dieser Erdsystemanalyse setzt Schellnhuber mit der Beschreibung einer zweiten kopernikanischen Wende ein. Auf die erste kopernikanische Wende als dem Übergang vom geo- zum heliozentrischen Weltbild zu Beginn der Neuzeit folge gegenwärtig eine zweite kopernikanische Revolution. Diese wende sich wieder der Erde zu, die als ein holisti-

17 H. J. Schellnhuber, „Earth system“ analysis and the second Copernican revolution, Nature 402 (2.12.1999), S. C19 (C22).

18 H. J. Schellnhuber/J. P. Kropp, Geocybernetics, Naturwissenschaften 85 (1998), S. 411 (423).

19 Schellnhuber, „Earth system“ analysis (Fn. 17), S. C22; vgl. auch ders., ebd., S. C20.

20 Vgl. W. C. Clark/P. J. Crutzen/H. J. Schellnhuber, Science for Global Sustainability, in: H. J. Schellnhuber/P. J. Crutzen/W. C. Clark/M. Claussen/H. Held (Hrsg.), Earth System Analysis for Sustainability, Cambridge 2004, S. 1 (6 ff.).

21 H. J. Schellnhuber/P. J. Crutzen/W. C. Clark/J. Hunt, Earth System Analysis for Sustainability, Environment 47/8 (10.10.2005), S. 11 (24).

22 Vgl. hierzu und zum Folgenden Schellnhuber, „Earth system“ analysis (Fn. 17), S. C19 ff.

23 Vgl. T. Hobbes, Leviathan, 3. Aufl., Frankfurt 1989, S. 5 ff., 94 ff.; zur politischen Ikonographie des Frontispiz von 1651 H. Bredekamp, Thomas Hobbes Der Leviathan, 3. Aufl., Berlin 2006, bes. S. $11 \mathrm{ff}$. 
sches „Erdsystem“ zu verstehen sei. Für die Analyse des Erdsystems E eröffnet Schellnhuber die folgende Gleichung:

$$
\begin{gathered}
\text { "E }=(\mathrm{N}, \mathrm{H}) \\
\text { where } \mathrm{N}=(\mathrm{a}, \mathrm{b}, \mathrm{c}, \ldots) ; \mathrm{H}=(\mathrm{A}, \mathrm{S}) .{ }^{“ 24}
\end{gathered}
$$

Das Erdsystem E auf der linken Seite der Gleichung entspricht einem Bereich von N und $\mathrm{H}$ auf der rechten Seite der Gleichung. ${ }^{25}$ Dabei steht $\mathrm{N}$ für die „Ökosphäre“, die sich selbst wiederum aus einer Vielzahl von komplex verbundenen planetarischen Untersphären - wie beispielsweise a (Atmosphäre), b (Biosphäre), c (Cryosphäre) etc. - zusammensetzt: $\mathrm{N}=(\mathrm{a}, \mathrm{b}, \mathrm{c}$...). Demgegenüber repräsentiert $\mathrm{H}$ den human factor, der wiederum zwei Subkomponenten aufweist: zum einen die physische Subkomponente A (Anthroposphäre), die das Aggregat allen individuellen menschlichen Lebens sowie aller individueller menschlicher Handlungen und Produkte darstellt; zum anderen die „,metaphysical' sub-component $S$ reflecting the emergence of a ,global subject'."26 Auf diese Weise wird das politische System in das Erdsystem einbezogen. Entscheidungen des globalen Subjekts S werden in der holistischen Perspektive der Erdsystemanalyse nicht außerhalb, sondern innerhalb des komplexen Interaktionsbereichs von Ökosphäre $\mathrm{N}$ und human factor $\mathrm{H}$ getroffen: $(\mathrm{N}, \mathrm{H})$.

Die Aufgabe des globalen Subjekts S ist die Regulierung des Erdsystems. ${ }^{27}$ Das globale Subjekt S repräsentiere - so Schellnhuber - „the collective action of humanity as a self-conscious control force that has conquered our planet. The global subject is real, although immaterial." ${ }^{28} \mathrm{~S}$ entstehe auf der Grundlage weltweiter Kommunikation als ein „direct global, polylogue' taking place via the Internet. Global telecommunication will ultimately establish a cooperative system generating values, preferences and decisions as crucial commonalities of humanity online." 29 Dabei ermögliche vor allem der Aufbau und die Anwendung eines Erdobservationssystems dem globalen Subjekt S, seine Identität auszubilden, seine eigenen ökologischen Fußabdrücke zu identifizieren und über digitale Erdmodellsimulationen kollektive, rationale Entscheidungen auf der globalen Ebene des Erdsystems zu treffen. Schließlich würden dicht vernetzte globale Institutionen sowie unzählige globale Netzwerke von Aktivisten den Resolutionen von S - wie beispielsweise internationalen Umweltkonventionen - zu ihrer Durchsetzung verhelfen. Hierin sieht Schellnhuber einen neuen demokratischen Verfassungsvertrag: „This is the emergence of a modern ,Leviathan', embodying teledemocracy and putting the seventeenth-century imagination of the

24 Schellnhuber, „Earth system“ analysis (Fn. 17), S. C20.

25 Vgl. hierzu und zum Folgenden Schellnhuber, „Earth system“ analysis (Fn. 17), S. C20 f.

26 Schellnhuber, „Earth system“ analysis (Fn. 17), S. C21.

27 Vgl. hierzu und zum Folgenden Schellnhuber, „Earth system“ analysis (Fn. 17), S. C22 f.

28 Schellnhuber, „Earth system“ analysis (Fn. 17), S. C22.

29 Schellnhuber, „Earth system“ analysis (Fn. 17), S. C22. 
English philosopher Thomas Hobbes into the shade. The global subject will reign over the centuries to come. One of its most responsible tasks will be to seek out a tolerable environmental future from the infinity of optional co-evolutions of $\mathrm{N}$ and A. In other words, S must guarantee sustainable development. “30

Für die Bestimmung dieser zentralen Aufgabe des globalen Subjekts S, eine nachhaltige Entwicklung für das gesamte Erdsystem zu gewährleisten, stellt Schellnhuber das Konzept einer „mathematical sustainable-development ethics“31 vor. Diese Ethik soll in einer zweidimensionalen „Theaterwelt“ („,theatre world“) ${ }^{32}$ darstellbar sein, in welcher der Entwicklungsraum P grundsätzlich durch alle denkbaren Möglichkeiten der Ko-Evolution der Ökosphäre N und der Anthroposphäre A gekennzeichnet ist:

$$
\text { „P }=(\mathbf{N}, \mathbf{A}) \text { “ } 33
$$

Es könnten - so Schellnhuber weiter - beispielsweise zunächst alle möglichen Formen der Ko-Evolution von globaler Durchschnittstemperatur einerseits und Weltsozialprodukt andererseits ermittelt werden. Für die Bestimmung einer nachhaltigen Entwicklung scheiden dabei zunächst alle pathologischen Optionen aus, die entweder zu einem für die Menschheit zu warmen oder zu kalten Erdsystem führen. Im Rahmen dieser Leitplanken ergeben sich sodann sehr unterschiedliche Möglichkeiten der Ko-Evolution von Öko- und Anthroposphäre, zwischen denen das globale Subjekt $S$ wählen kann. Das Erdmanagement des globalen Subjekts $S$ kann sich dabei an fünf Paradigmen orientieren: ${ }^{34}$ erstens Standardization, die einen langfristigen Korridor der Ko-Evolution von Ökosphäre $\mathrm{N}$ und Anthroposphäre A beschreibt; zweitens Optimization, die auf die Maximierung einer ko-evolutiven Wohlfahrtsfunktion von Ökosphäre $\mathrm{N}$ und Anthroposphäre A zu einem bestimmten Zeitpunkt zielt, beispielsweise in Form eines gleichheitssensiblen Re-Designs von geostrategischer Arbeitsteilung, Energieerzeugung und Biodiversität; drittens Pessimization, die Mindeststandards und Mindestsicherheiten gewährleistet, um zum Beispiel im Klimaschutz katastrophale Entwicklungen zu vermeiden; viertens Equitization, die darauf gerichtet ist, Optionen für künftige Generationen zu bewahren; und fünftens Stabilization, die den „N-A complex“ ${ }^{35}$ in einen gewünschten Zustand der Ko-Evolution bringt und dort hält, beispielsweise durch Geoengineering.

Es mag zugleich auf der ko-evolutiven Linie dieser Langzeitperspektive liegen, dass Schellnhuber - zusammen mit Paul Crutzen, William Clark und Julian Hunt-in der

30 Schellnhuber, „Earth system“ analysis (Fn. 17), S. C22 f.

31 Schellnhuber, „Earth system“ analysis (Fn. 17), S. C23.

32 Vgl. hierzu und zum Folgenden Schellnhuber, „Earth system“ analysis (Fn. 17), S. C22.

33 Schellnhuber, „Earth system“ analysis (Fn. 17), S. 22.

34 Vgl. hierzu und zum Folgenden Schellnhuber, „Earth system“ analysis (Fn. 17), S. C23.

35 Schellnhuber, „Earth system“ analysis (Fn. 17), S. C23. 
Fortentwicklung seines Ansatzes das Selbstverständnis des Menschen in einer postanthropozänen Periode relativiert sieht. ${ }^{36}$ In Abgrenzung zu Stanisław Lems Solaris-Vision sollen Bio-, Info- und Geosphäre zwar nicht zu einer planetarischen Einheit verschmelzen, ${ }^{37}$ sondern in ihrer ko-evolutiven Entwicklung weiterhin unterscheidbar bleiben. Dennoch ändert sich die Charakterisierung des globalen Subjekts im Erdsystem, wenn dieses nun als ein „post-Anthropocene Leviathan representing humanity’s collective self-conscious willpower" ${ }^{38}$ verstanden wird. Dieser post-anthropozäne Leviathan soll vor der Wahl stehen, das Erdsystemmanagement an den Konzepten des „planetary design“, der „pure auto-evolution“ oder der „controlled coevolution“ auszurichten. ${ }^{39}$ Dabei ist „planetary design“ auf die Ergänzung der Ökosphäre nach menschlichen Bedürfnissen durch Geoengineering und Nanotechnologie ausgerichtet. „Pure auto-evolution“ zielt auf die Anpassung der Menschheit an Umweltveränderungen, wobei etwa an gentechnische Eingriffe zu denken ist. Demgegenüber setzt „controlled coevolution“ darauf, dass die Menschheit sich selbst, die Natur und ihr beiderseitiges Verhältnis auf der Grundlage feststehender Prinzipien ständig neu erfindet. Schellnhuber et al. favorisieren im Ergebnis eine Mischung aus den beiden zuletzt genannten Steuerungsansätzen: „Sustainable autoevolution " 40 soll Bewahrung und Innovation, Natur und Zivilisation sowie Anpassungsfähigkeit („responsiveness“) und Autonomie angemessen ausbalancieren. Das post-anthropozäne Moment dieser Formel von der „sustainable auto-evolution“ liegt darin, dass die Menschen ihre geologische Gestaltungsmacht, die ihrem Erdzeitalter ihren Namen gibt, wieder relativieren, indem sie sich stärker auf die Ökosphäre einlassen. Das von ihnen konstituierte globale Subjekt entwickelt sich so vom „modernen Leviathan“ zum „post-anthropozänen Leviathan“. In dieser Metamorphose des globalen Subjekts S verändert sich nicht die politische Funktion, wohl aber die evolutionäre Sensibilität des Leviathan im Erdsystem.

\section{2. „Große Transformation“}

Diese erdsystemanalytische Integration des globalen Leviathan ist keineswegs „nur“ Theorie geblieben, sondern prägt maßgeblich den Gesellschaftsvertrag für eine Große Transformation, den der WBGU unter dem Vorsitz Hans Joachim Schellnhubers im Jahr 2011 vorgelegt hat: ${ }^{41}$ In seiner Konturierung eines „Social Contract

36 Vgl. hierzu und zum Folgenden Schellnhuber/Crutzen/Clark/Hunt, Earth System Analysis (Fn. 21), S. 24.

37 Vgl. S. Lem, Solaris, 7. Aufl., Berlin 2014, S. 32 ff., 102 f.

38 Schellnhuber/Crutzen/Clark/Hunt, Earth System Analysis (Fn. 21), S. 24.

39 Vgl. hierzu und zum Folgenden Schellnhuber/Crutzen/Clark/Hunt, Earth System Analysis (Fn. 21), S. 24.

40 Schellnhuber/Crutzen/Clark/Hunt, Earth System Analysis (Fn. 21), S. 24.

41 Vgl. WBGU, Welt im Wandel. Gesellschaftsvertrag für eine große Transformation, Berlin 2011 (http:// www.wbgu.de/fileadmin/templates/dateien/veroeffentlichungen/hauptgutachten/jg2011/wbgu_jg2011.pdf [Abruf: 19.2.2013]). 
for Sustainability “42 geht auch der WBGU von der geo- und ökologischen Bestandsaufnahme aus, dass die Erde in das Anthropozän eingetreten ist. ${ }^{43}$ Gerade mit Blick auf Artensterben, Klimawandel und Ressourcenverbrauch sei der Mensch „ohne Zweifel eine dominierende Kraft innerhalb des Erdsystems geworden. "44 Dabei beschreibe - so der WBGU weiter - das Anthropozän „,nicht nur die vom Menschen verursachte Wirkung auf das Erdsystem, sondern auch den kognitiven Wandel der globalen Zivilisation, die sich ihrer Bedeutung als formende Kraft zunehmend bewusst wird. “45 Ein verantwortungsvolles Handeln müsse deshalb die „planetarischen Leitplanken " 46 einhalten. Diese definierten den geo- und ökologischen Rahmen, innerhalb dessen Entwicklung und Fortschritt auf nachhaltige Weise verlaufen könnten. Deshalb sei eine „Große Transformation“ notwendig, deren Eingriffstiefe der neolithischen und der industriellen Revolution als den beiden fundamentalen Umbrüchen in der Menschheitsgeschichte entspreche. Die „Große Transformation“ umfasse „tiefgreifende Änderungen von Infrastrukturen, Produktionsprozessen, Regulierungssystemen und Lebensstilen sowie ein neues Zusammenspiel von Politik, Gesellschaft, Wissenschaft und Wirtschaft. “" ${ }^{47}$ Die Umsetzung dieser „Großen Transformation“ erfordere eine neue soziale „Geschäftsgrundlage“, die der WBGU als einen „neuen Weltgesellschaftsvertrag für eine klimaverträgliche und nachhaltige Weltwirtschaftsordnung “48 formuliert. Dieser globale Gesellschaftsvertrag für Nachhaltigkeit solle dabei weniger auf dem Papier als vielmehr im Bewusstsein der Menschen verankert sein. ${ }^{49}$ Mit ihm bringe die Menschheit zum Ausdruck, dass sie „kollektive Verantwortung für die Vermeidung gefährlichen Klimawandels und anderer planetarischer Risiken übernimmt. “50 Der Vertrag basiere auf der Idee, „dass Individuen und die Zivilgesellschaften, die Staaten und die Staatengemeinschaft sowie die Wirtschaft und die Wissenschaft kollektive Verantwortung für die Vermeidung gefährlichen Klimawandels und für die Abwendung anderer Gefährdungen der Menschheit als Teil des Erdsystems übernehmen. " ${ }^{51}$ Auf diese Weise kombiniere der globale Sozialkontrakt „eine Kultur der Achtsamkeit (aus ökologischer Verantwortung) mit einer Kultur der Teilhabe (als demokratische Verantwortung) sowie mit einer Kultur der Verpflichtung gegenüber zukünftigen Generationen (Zukunftsver-

42 So die - sehr viel prägnantere - englischsprachige Übersetzung: WBGU, World in Transition. A Social Contract for Sustainability, Berlin 2011 (http://www.wbgu.de/fileadmin/templates/dateien/veroeffentlichungen/hauptgutachten/jg2011/wbgu_jg2011_en.pdf [Abruf: 19.2.2013]).

43 Vgl. hierzu und zum Folgenden WBGU, Welt (Fn. 41), S. 33 f., 66, 98, 288, 415.

44 WBGU, Welt (Fn. 41), S. 33.

45 WBGU, Welt (Fn. 41), S. 33.

46 WBGU, Welt (Fn. 41), S. 34, 66, 288.

47 WBGU, Welt (Fn. 41), S. 2; vgl. auch ders., ebd., S. 23.

48 WBGU, Welt (Fn. 41), S. 2; vgl. ders., ebd., S. 8.

49 Vgl. WBGU, Welt (Fn. 41), S. 2.

50 WBGU, Welt (Fn. 41), S. 8.

51 WBGU, Welt (Fn. 41), S. 2; vgl. ders., ebd., S. 8. 
antwortung). “52 Als zentralen Akteur dieses globalen Vertragssystems weist der WBGU den „gestaltenden Staat“ ${ }^{\text {53 }}$ aus, der aktiv Prioritäten für die „Große Transformation “ setze, die Partizipationsmöglichkeiten für die Bürgerinnen und Bürger erweitere und der Wirtschaft nachhaltige Handlungsoptionen eröffne. Auf globaler Ebene schlägt der WBGU die Schaffung eines „UN-Rates für Nachhaltige Entwicklung “ 54 vor, der dem Weltsicherheitsrat ebenbürtig sein soll.

\section{Konzeptkritik}

Der neue Weltgesellschaftsvertrag für Nachhaltigkeit formuliert ein sozial, politisch und normativ umfassendes Konzept, das auf drei Grundpfeilern ruht: erstens der Anwendung der Metapher des Sozialvertrags auf die Weltgesellschaft, zweitens der Fixierung auf das Prinzip der Nachhaltigkeit und drittens dem holistischen Transformationsmodus der „Gestaltung “. Doch alle drei Bausteine dieser politischen Neuordnung des Anthropozän begegnen konzeptionellen Einwänden.

\section{Weltgesellschaftsvertrag}

Der Sozialvertrag ist wohl das klassischste aller Instrumente, um der politischen Inklusion einer Gesellschaft normativen Ausdruck zu verleihen. ${ }^{55}$ Dabei ist es sicherlich zu begrüßen, dass die anthropozänen Variationen des Gesellschaftsvertrags im Vergleich mit ihren tradierten Vorgängern und Vorbildern ökologische Belange zentral berücksichtigen. Doch gleichzeitig wirft dieses kontraktualistische AnthropozänKonzept die Frage auf, ob das überkommene Modell des Gesellschaftsvertrags überhaupt einen angemessenen Rahmen für das normative Selbstverständnis einer kulturell, politisch, religiös und wirtschaftlich fragmentierten Weltgesellschaft mit ihren äußerst differenzierten Perzeptionen von „Natur“ bieten kann. Damit soll nicht gesagt werden, dass der Sozialvertrag als normative Inklusionsmetapher einer Gesellschaft ausgedient hätte. Er mag weiterhin die politische Selbstbeschreibung sowie das normative Selbstverständnis von staatlich verfassten Gesellschaften zum Ausdruck bringen und in dieser Funktion auch als Erklärungs- bzw. Legitimationsfragment in supra- und internationale Verbünde sowie in die Vernetzungen der Weltgesellschaft integrierbar sein. Der Sozialvertrag ist jedoch als Konzept politisch zu einseitig, kulturell zu undifferenziert und - insbesondere - normativ zu überdeterminierend, um einen politischen Rahmen für die Weltgesellschaft zu bilden. Dieser normative overstretch wird deutlich, wenn man zwischen der Begründung und der Substantiierung einer Globalisierung des Sozialvertragsmodells unterscheidet: Die Entstehung des globalen politischen Körpers wird damit begründet, dass die Menschen durch Umwelteingriffe eine globale Natur schaffen, welche wiederum die Exis-

52 WBGU, Welt (Fn. 41), S. 2; vgl. ders., ebd., S. 8, 282, 294.

53 WBGU, Welt (Fn. 41), S. 2; vgl. ders., ebd., S. 7 ff., 114 f., 215 ff.

54 WBGU, Welt (Fn. 41), S. 2; vgl. ders., ebd., S. 20, 335.

55 Vgl. M. Loughlin, Foundations of Public Law, Oxford 2010, S. 108 ff., 278 ff. 
tenz der Menschheit bedroht. ${ }^{56}$ Doch diese abstrakte Begründung der Entstehung eines globalen politischen Körpers ist das eine, seine konkrete Substantiierung in der Erlebnis- und Erfahrungswelt der Menschen das andere. Während Thomas Hobbes mit seiner Metapher des „Krieg[s] eines jeden gegen jeden“ ${ }^{57}$ seit fast einem halben Jahrtausend auf den historischen Evidenzappell setzen kann, dass im Zweifel der Nachbar der natürliche Feind des Menschen ist, fehlt es (bislang) an einer entsprechenden Verankerung der ökologischen Bedrohung im individuellen Gefühls- und politischen Erfahrungshaushalt der Menschen: $\mathrm{Zu}$ unsicher sind die ökologischen Wirkungszusammenhänge von Kultur und Natur, zu abstrakt die Selbstbetroffenheit durch die ökologischen Folgen individuellen und kollektiven Handelns. Hierin mag auch der Grund liegen, dass Schellnhuber die wissenschaftliche Feststellung der ökologischen Gefährdungen über die Erdsystemanalyse außer Streit stellt, um die Substantiierung des globalen politischen Körpers in einem ganz anderen Bereich zu suchen: der Idee einer digital vernetzten pluralistischen Weltgesellschaft, durch die das neue globale Subjekt $S$ kommunikativ konstituiert wird. Diesen globalen Netzdiskursen wird von Schellnhuber die Fähigkeit zugeschrieben, einerseits Werte, Präferenzen und Entscheidungen zu generieren und andererseits im Zusammenspiel von internationalen Organisationen und weltweit vernetzten Aktivisten die Entscheidungen des globalen Subjekts S durchzusetzen. Auf diese Weise wird die Herrschaft des „modernen Leviathan“ als eine „Teledemokratie“ legitimiert: „Why not turn to ,cyberspace democracy', where the actors and stake-holders in the coevolution process grind out crucial decisions via interactive planning games based on Earth System modelling? This would ensure a broad distribution of responsibility through equitable access to knowledge, if not wisdom. "58 In der heutigen Rückschau auf diese Argumentation aus dem Jahr 1999 fällt der idealistische Vorschuss auf, mit dem das Netz im Hinblick auf Werte, Entscheidungen und Demokratie bedacht wurde und teilweise immer noch bedacht wird. ${ }^{59}$ Auch wenn man die Entwicklung der Sozialen Medien seither nicht als eine Verlustgeschichte demokratischer Öffentlichkeit beschreiben will, haben die Erfahrungen mit dem Web 2.0 im vergangenen Jahrzehnt gezeigt, dass die Netzkultur keineswegs gemeinsame Werte, kollektive Entscheidungen und freiheitliche Demokratie gleichsam automatisch aus sich heraus generiert. ${ }^{60}$ Damit ist nicht gesagt, dass das Internet keine Rolle für die Kommunikation einer globalen Gesellschaft und gerade auch ihrer ökologischen Probleme spielt. Doch die politische Netzkultur muss erst noch ihre Verfassung und vor allem auch

56 Vgl. M. Serres, Der Naturvertrag, Frankfurt 1994, S. 75.

57 Hobbes, Leviathan (Fn. 23), S. 97 (Klammerzusatz durch den Verfasser).

58 H. J. Schellnhuber, Discourse: Earth System Analysis, in: ders./V. Wenzel (Hrsg.), Earth System Analysis, Berlin/Heidelberg/New York 1998, S. 3 (183).

59 Vgl. nur W. Plaum, Die Wiki-Revolution, Berlin 2012, S. $147 \mathrm{ff}$.

60 Vgl. C. Sunstein, Repulic.com 2.0, Princeton/Oxford 2007, S. 46 ff., 138 ff. 
Anschluss an die demokratischen Institutionen der Offline-Welt finden. ${ }^{61}$ Schellnbubers „Leviathan“ kann 1999 zeitbedingt allenfalls die Anfänge dieser Komposition einer (globalen) Öffentlichkeit reflektieren. Er substantiiert allerdings nicht, in welchen neuen Strukturen sich dieser Wandel vollzieht.

Der WBGU sieht die Fragmentierung der politischen Welt durchaus: ${ }^{62}$ den Pluralismus der Weltgesellschaft(en) und der globalen Kulturen, das politische Mehrebenensystem mit seinen Verfassungen, Verbünden und Vernetzungen, den politischen Einfluss von Zivilgesellschaft, transnationalen Unternehmen und NGOs, das Orchester von internationalen Organisationen und hybriden Rechtsregimen. ${ }^{63}$ Doch gerade dies wirft die Frage auf, wie der WBGU diese Realitäten sehen, aber dennoch am holistischen Konzept eines Weltgesellschaftsvertrags festhalten kann, um die "Große Transformation“ der Weltsysteme politisch zu rahmen. Eine Erklärung dafür könnte sein, dass der WBGU einer politischen Versuchung erlegen ist, auf die Bruno Latour kritisch hingewiesen hat: ${ }^{64}$ Sie besteht darin, auf das Anthropozän als eine globale Herausforderung mit der Konstitution eines „Anthropos“ 65 im Sinn eines globalen politischen Körpers der Menschheit zu reagieren. Die problematischen Folgen dieser holistischen Konzeption bleiben nicht auf die politische Theorie beschränkt, sondern schlagen sich auch in politischer Praxis und rechtlicher Governance nieder: Gerade wenn der WBGU den neuen Gesellschaftsvertrag für Nachhaltigkeit im Bewusstsein jedes einzelnen Menschen verankert sehen möchte, werden die Bürgerinnen und Bürger globale Antworten auf die anthropozäne Herausforderung erwarten, die bisher ausgeblieben sind und in einer fragmentierten Weltgesellschaft wohl auch künftig politisch nicht realisierbar sein werden.

\section{Nachhaltigkeit}

Das Prinzip der nachhaltigen Entwicklung ist auf einen angemessenen Ausgleich sozialer, ökonomischer und ökologischer Interessen gerichtet. ${ }^{66}$ Es war und ist zweifellos revolutionär, dass sich der Nachhaltigkeitsgrundsatz in den letzten zwanzig Jahren zu einem der zentralen Prinzipien des nationalen, europäischen und internationalen Umweltrechts mit Ausstrahlung auf fast alle anderen Rechtsgebiete entwickelt hat. ${ }^{67}$ Andererseits darf aber auch nicht übersehen werden, dass es sich bei

61 Vgl. J. Kersten, Transformationen der Demokratie, München 2014, S. 15 ff.: ders., Schwarmdemokratie, JuS 2014, S. $673 \mathrm{ff}$.

62 Vgl. WBGU, Welt (Fn. 41), S. 185 ff.

63 Vgl. G. Teubner, Verfassungsfragmente, Berlin 2012, S. $72 \mathrm{ff}$.

64 Vgl. B. Latour, The Anthropocene and the destruction of the image of the Globe. Gifford Lecture Series „Facing Gaia. A new enquiry into Natural Religion“. 4. Lecture, University of Edinburgh, 25.2.2013 (http://www.youtube.com/watch?v=4-16FQN4P1c [Abruf: 30.7.2013]).

65 Latour, Anthropocene (Fn. 64); ferner aber ders., Wir sind nie modern gewesen, Frankfurt 2008, S. 182.

66 Vgl. R. Schmidt/W. Kahl, Umweltrecht, 8. Aufl., München 2010, $\mathbb{1}$, Rdnr. 29 ff.

67 Vgl. grundlegend W. Kahl (Hrsg.), Nachhaltigkeit als Verbundbegriff, Tübingen 2008. 
der Nachhaltigkeit um einen normativ sehr nachgiebigen und konservativen Grundsatz handelt. Die normative Nachgiebigkeit des Nachhaltigkeitsprinzips haben $\mathrm{Mi}$ chael Krautzberger und Bernhard Stüer mit dem Verweis auf dessen „theatralische Sendung " veranschaulicht: Ökologische Belange sind im Verhältnis zu sozialen und wirtschaftlichen Interessen problemlos wegwägbar, solange die dabei vergossenen Krokodilstränen nur groß genug sind. ${ }^{68}$ Dieser Einwand spitzt sich im Zuge einer zunehmenden Ökonomisierung der sozialen Welt immer stärker zu. Noch schwerer wiegt aber der konservative Einschlag des Prinzips der nachhaltigen Entwicklung: ${ }^{69}$ Der Nachhaltigkeitsgrundsatz ist auf einen bewahrenden Interessenausgleich gerichtet. Er muss also in allen Fällen versagen, in denen eine Entwicklung durch eine abwägende Steuerung nicht mehr möglich ist. Was meint nachhaltige Entwicklung angesichts des weltweiten Kollapses von Biodiversität? Die menschliche Ausrottung der Arten beschleunigt sich immer mehr, und bis zum Ende dieses Jahrhunderts könnten die Hälfte der derzeit noch existierenden Arten verschwunden sein. ${ }^{70} \mathrm{Im}$ Hinblick auf die Biodiversität befinden wir uns also längst in einer nach-nachhaltigen Entwicklung und haben selbst Probleme, auch nur ansatzweise von Resilienz oder Resistenz zu sprechen. ${ }^{71}$ Was meint darüber hinaus Nachhaltigkeit hinsichtlich der globalen Erfahrung mit atomaren Katastrophen, für die Three Miles Island, Tschernobyl und Fukushima stehen?72 Auch mit Blick auf die „Metaphysik der Endlagerung " 73 von atomaren Abfällen, die noch über Jahrhunderte strahlen werden, lässt sich kaum etwas Nachhaltiges sagen, sondern letztlich nur der Gefahrenbegriff reaktivieren. Damit ist nicht gemeint, dass der Nachhaltigkeitsgrundsatz bedeutungslos wäre oder insgesamt verabschiedet werden müsste. ${ }^{74}$ Doch als holistisches Grundprinzip eines Weltgesellschaftsvertrags ist er nicht in der Lage, die ökologischen Probleme des Anthropozän angemessen abzubilden. Dafür ist das Nachhaltigkeitsprinzip einerseits normativ zu schwach und andererseits von seinem effektiven Anwendungsbereich zu unterkomplex. Deshalb lautet eine der spannendsten Fragen des Anthropozän: Was kommt nach der Nachhaltigkeit?

\section{3. "Gestaltung“}

Die holistischen Modelle Schellnhubers und des WBGU setzen auf Management, Regulierung und Kontrolle bzw. auf das Leitbild des „gestaltenden Staats“, wenn es um die Umsetzung des neuen Weltgesellschaftsvertrags für Nachhaltigkeit geht. Doch gerade der Begriff „Gestaltung“ entwickelt eine biopolitisch vielschichtige Be-

68 Vgl. M. Krautzberger/B. Stüer, Städtebaurecht 2004, DVBl. 2004, S. 914 (924).

69 Vgl. P. Sloterdijk, Zeilen und Tage, Berlin 2012, S. 490 f.

70 Vgl. U. K. Heise, Nach der Natur, Berlin 2010, S. 9; J. Kersten, „Im Dickicht der Städte“, UPR 2012, S. $15 \mathrm{ff}$.

71 Vgl. zu Resistenz unten D.II.1; zu Resilienz unten D.II.2.

72 Vgl. R. Spaemann, Nach uns die Kernschmelze, Stuttgart 2011, S. $7 \mathrm{ff}$.

73 P. Sloterdijk, Zorn und Zeit, Frankfurt 2006, S. 146.

74 Vgl. unten D.II.3. 
deutung, wenn im anthropozänen Drift die überkommene Unterscheidung zwischen Natur und Kultur zu verschwimmen beginnt: Schon heute sind der überwiegende Teil der globalen Ökosysteme und Landschaften nicht mehr in einem ursprünglichen Sinn „natürlich“. Vielmehr wurden und werden sie von Menschen beeinflusst, gestaltet und designt. Planet Gardening durch „Weltgärtner“ ist zu einem zentralen gestaltungsbezogenen Handlungsmotiv des Anthropozän geworden. ${ }^{75} \mathrm{Nach}$ der Auffassung von Reinhold Leinfelder gehört es zu den anthropozänen Grundeinsichten, dass wir nicht mehr zwischen der „guten“ bewahrenswerten Natur und der „bösen“ menschlichen Technik unterscheiden können: ${ }^{76}$ Natur und Kultur seien Teil eines Gesamtsystems, das so gestaltet werden müsse, damit es nicht aus dem Gleichgewicht gerate. Auf diese Weise erscheint eine anthropozäne Cultura ganz in der lateinischen Bedeutung dieses Worts möglich, wenn eine kultivierende Gestaltung der Natur im „Rambunctious Garden“ einer „Post-Wild World“ befürwortet wird. ${ }^{77}$ Diese Konzepte von „Kultur-Natur“78 bzw. „Naturecultures“79 verdeutlichen, warum „Gestaltung “ in diesem Anthropozän-Konzept letztlich weit über die schlichte Werbung für eine aktive, demokratische und zivilgesellschaftliche Umweltpolitik hinausgeht und der „Imperativ des Gestaltens" 80 auch Formen bionischer, bioökonomischer und biotechnischer Reformansätze transzendiert. ${ }^{81}$ Die anthropozäne Cultura steht eher in der Denktradition einer „Naturdämmerung“, 82 in der sich die Menschen in einer „entgöttlichten Natur“ selbst „vernatürlichen“. ${ }^{83}$ Der Katalysator dieser Vernatürlichung ist die technische Gestaltung des changierenden Spannungsverhältnisses von Natur und Kultur, was sich in umstrittenen Projekten wie Geoengineering oder Synthetic Biology widerspiegelt: So schlägt Crutzen selbst großangelegte Geoengineering-Forschungsprojekte zur „Optimierung“ des Klimas vor. ${ }^{84}$

\section{Konzeptdimensionen}

Der globale Gesellschaftsvertrag für Nachhaltigkeit, wie er von Schellnhuber und dem WBGU mit besonderem Fokus auf die Bewältigung des Klimawandels vorge-

75 Vgl. R. Leinfelder, Wir Weltgärtner, Die Zeit, 10.1.2013 (http://www.zeit.de/2013/03/AnthropozaenProjekt-Berlin-HKW-Leinfelder/komplettansicht?print=true [Abruf: 19.2.2013]); auch Schwägerl, Menschenzeit (Fn. 2), S. 112, 120, 137, 349, 351.

76 Vgl. hierzu und zum Folgenden Leinfelder, Weltgärtner (Fn. 75); ferner Schwägerl, Menschenzeit (Fn. 2), S. 13.

77 Vgl. E. Marris, Rambunctious Garden, New York 2011.

78 Markl, Natur (Fn. 6), S. 334.

79 D. Haraway, The Companion Species Manifesto, Chicago 2003, S. 1.

80 Schwägerl, Menschenzeit (Fn. 2), S. 101; vgl. auch ders., ebd., S. 128.

81 Vgl. Leinfelder, Weltgärtner (Fn. 75); Schwägerl, Menschenzeit (Fn. 2), S. 156 ff., 196, 321, 358.

82 Markl, Natur (Fn. 6), S. 324.

83 Vgl. F. Nietzsche, Fröhliche Wissenschaft, in: ders., Werke, 2. Bd., 2. Aufl., München 1958, S. 7 (116 [Nr. 109]).

84 Vgl. Crutzen, Geology (Fn. 1), S. 23; krit. Leinfelder, Weltgärtner (Fn. 75); Schwägerl, Menschenzeit (Fn. 2), S. 299. 
schlagen wird, verbindet die vier Dimensionen des Anthropozän-Konzepts - Zeit, Raum, Akteur und Wissenschaft ${ }^{85}$ - zu einem holistischen Modell: Die Zeitdimension wird zunächst durch den Zeitdruck des Klimawandels bestimmt, geht aber sodann in eine dauerhafte menschliche Nachhaltigkeitsära über. Mit diesem nachhaltigen Ende der Geschichte in einem „langen Jetzt" ${ }^{\text {86 }}$ verliert die Zeit in diesem holistischen Konzept des Anthropozän letztlich ihre politische Bedeutung: Politik und Recht sind stets auf eine nachhaltige Gegenwart und immer nachhaltigere Zukunft bezogen.

Die zentrale politische Perspektive dieses Vertragsmodells ist deshalb nicht die Zeit, sondern der Raum: Auch die Raumperspektive wird zunächst ganz durch die Klimafixierung des Modells bestimmt und durch das Motiv der „planetarischen Leitplanken “ weiter unterstrichen: Der Klimawandel versinnbildlicht die Limits des Planeten als geschlossenen, sich aufheizenden Raum, in welchem der Menschheit das Atmen zunehmend schwerfällt. Die Klaustrophobie dieses Raumkonzepts kommt in Anlehnung an Richard Buckminster Fuller ${ }^{87}$ - in der Metapher zum Ausdruck, dass das Raumschiff Erde über keinen Notausgang verfüge. ${ }^{88}$ Es ist diese Klaustrophobie, die den politischen Handlungsdruck des kontraktualistischen AnthropozänKonzepts erzeugt. Sie bringt die Menschen dazu, den globalen Gesellschaftsvertrag zu schließen und sich selbst in Form der „Menschheit“ als zentralen politischen Akteur des Anthropozän zu konstituieren. Dabei steigert das mit dem Raumkonzept der „aufgeheizten Erde“ einhergehende Gefühl der Künstlichkeit der Welt die wissenschaftlich vermittelten Gestaltungsoptionen: ${ }^{89}$ Die Unterscheidung von Natur und Kultur verschwimmt in einer gestaltbaren anthropozänen Cultura. In dieser kommt es darauf an, die sozialen, ökonomischen und ökologischen Interessen nachhaltig auszugleichen. Dementsprechend geht es der „Großen Transformation“ letztlich darum, soziale, ökonomische und ökologische Systeme innerhalb der „planetarischen Leitplanken“ gleichsam „ko-immun“ auszubalancieren. Die Konzepte, die wiederum diese Idee eines ökologisch inspirierten „Ko-immunismus“90 aufnehmen, mögen ganz bewusst mit ihrem ideengeschichtlichen Assoziations- und Dissoziationspotenzial zum Kommunismus spielen. ${ }^{91}$ Doch zugleich spiegelt sich in dieser „ko-

85 Vgl. oben A.II.

86 Schwägerl, Menschenzeit (Fn. 2), S. 14, 79, $311 \mathrm{ff}$.

87 Vgl. R. B. Fuller, Bedienungsanleitung für das Raumschiff Erde und andere Schriften, Reinbek 1973.

88 Vgl. P. J. Crutzen/M. Davis/M. D. Mastrandrea/S. H. Schneider/P. Sloterdijk, Das Raumschiff Erde hat keinen Notausgang. Energie und Politik im Anthropozän, Berlin 2011; ferner Markl, Natur (Fn. 6), S. 342.

89 Vgl. zur artifiziellen Perzeption der sich erwärmenden Erde Schwägerl, Menschenzeit (Fn. 2), S. 9 ff.

90 P. Sloterdijk, Du mußt dein Leben ändern, Frankfurt 2009, S. 711; ferner ders., Zeilen (Fn. 69), S. 337, 371; ders., Ausgewählte Übertreibungen, Berlin 2013, S. 343 f., 381 f.

91 Sloterdijk, Leben (Fn. 90), S. 713; zurückhaltender ders., Wie groß ist „groß“?, in: Crutzen/Davis/ Mastrandrea/Schneider/Sloterdijk, Raumschiff (Fn. 88), S.106; ders., Übertreibungen (Fn. 90), S. $343 \mathrm{f} ., 381 \mathrm{f}$. 
immunen“ Vorstellung einer nachhaltigen Ausdifferenzierung der sozialen, ökologischen und ökonomischen Subsysteme im geschlossenen Erdsystem eine Grundkonstante raumpolitischen Denkens. Auf diese hat Werner Sombart 1906 im Rahmen seiner Antwort auf die Frage Warum gibt es in den Vereinigten Staaten keinen Sozialismus? mit der Möglichkeit der „Flucht des Arbeiters in die Freiheit“92 hingewiesen: Ohne offene Grenze - ohne Frontier - introvertieren soziale, politische und wirtschaftliche Systeme. Sicherlich gilt es zwischen der historischen Wirklichkeit, der kulturellen Erzählung, der wissenschaftlichen These und der metaphorischen Fassung der Frontier zu unterscheiden. ${ }^{93}$ Doch das von Sombart beschriebene Phänomen der Freiheit durch Flucht lässt sich auch als allgemeine Freiheitsidee fassen, nicht nur räumlich, sondern in jeder Hinsicht aus Systemen auszubrechen: Dinge anders zu sehen, anders zu fühlen, anders zu denken, anders zu leben. Die gesellschaftlichen Freiheits- und Innovationsverluste, die mit dem Entwurf des globalen Gesellschaftsvertrags für eine Große Transformation verbunden sind, hat Carl Christian von Weizsäcker auf den Punkt gebracht: „Der gestaltende Staat für eine Politik aus einem Guss! Karl Popper und Friedrich August von Hayek würden sich im Grabe umdrehen, wenn sie dies als hochoffizielles Rezept für eine neue Form der Demokratie zu hören bekämen. “94 Poppers Freiheit der institutionalisierten Revisionen ${ }^{95}$ und von Hayeks Freiheit der spontanen Ordnungen ${ }^{96}$ sind Entdeckungsverfahren, die nicht nur politisch und ökonomisch, sondern vor allem auch ökologisch funktionieren. Diese Entdeckungsverfahren realisieren sich jedoch vor allem über die Freiheit, ExitOptionen zu nutzen, und auf diese Weise über neue Frontiers neue Intelligenz-, Konflikt- und Wissensräume für das Anthropozän zu erschließen. ${ }^{97}$ Die holistischen Vertragskonzepte des Anthropozän lassen diese sozialen, ökonomischen und ökologischen Potenziale jedoch ungenutzt.

\section{Komposition}

In seiner Vorlesung The Anthropocene and the destruction of the image of the Globe an der Universität Edinburgh vom 25. Februar 2013 hat Bruno Latour seine

92 W. Sombart, Warum gibt es in den Vereinigten Staaten keinen Sozialismus?, Tübingen 1906, S. $134 \mathrm{ff}$.

93 Vgl. C. Mauch, Mensch und Umwelt, München 2014, S. 39 f.

94 C. C. $v$. Weizsäcker, Die große Transformation: ein Luftballon, FAZ, 30.9.2011, S. 12.

95 Vgl. K. R Popper, Die offene Gesellschaft und ihre Feinde 1, 6. Aufl., Tübingen 1980, S. 95 f., 174 ff., 201, 227, $267 \mathrm{f}$.

96 Vgl. F. A. v. Hayek, Die schöpferische Kraft einer freien Zivilisation, in: ders., Gesammelte Schriften, Band A4, Tübingen 2003, S. 31 ff.; ders., Der Wettbewerb als Entdeckungsverfahren, ebd., S. $132 \mathrm{ff}$.

97 Vgl. Sloterdijk, Ausgewählte Übertreibungen (Fn. 90), S. 42 f., zum utopischen Potenzial eines „Intelligenzraumes". 
kompositionistische Sicht des neuen Erdzeitalters entwickelt, ${ }^{98}$ indem er an seinen Versuch eines ,Kompositionistischen Manifests ${ }^{\circ 9}$ anknüpft.

\section{I. „Kompositionistisches Manifest“}

In seiner Edinburgher Anthropocene-Lecture entfaltet Latour ein kompositionistisches Konzept des Anthropozän. ${ }^{100}$ In diesem Zusammenhang weist er zunächst auf den epistemologischen Coup der Naturwissenschaften hin: Während die Geisteswissenschaften bereits in die „Posthumien“101 aufgebrochen seien, stellten die Naturwissenschaften den Menschen (wieder) ganz in den erdpolitischen Mittelpunkt, wenn sie das Anthropozän ausriefen. ${ }^{102}$ Zugleich bezweifelt Latour jedoch, dass ein Anthropozän-Konzept (normativ) stark genug sein könnte, um die Menschheit - in Form eines „Anthropos“ - als zentralen Akteur des Anthropozän politisch zu konstituieren. Gerade dieser letzte Punkt verdeutlicht zugleich, worum es Latour selbst mit seinem kompositionistischen Theorieansatz geht: Es gibt keine vorgegebenen politischen, religiösen, natürlichen oder wissenschaftlichen Einheiten. Vielmehr müssen die anthropozänen Akteur-Netzwerke erst neu zusammengesetzt, d.h. komponiert werden. ${ }^{103}$ "The notion of the Globe“ - so Latour in seiner AnthropoceneLecture - „and any global thinking entail the immense danger of unifying too fast what should be composed instead. "104 Damit nimmt Latour auf seine Variation der Akteur-Netzwerk-Theorie Bezug, die sich in einer Vernetzung von menschlichen und nichtmenschlichen Akteuren bzw. Aktanten entfaltet ${ }^{105}$ und wendet diese auf die neue Erdepoche an: Wie im Titel seiner Vorlesung angekündigt, de- und rekomponiert er zunächst „the image of the Globe“. Die Erde wird als Globus dekomponiert, um - im Rückgriff auf Peter Sloterdijks Sphärenprojekt ${ }^{106}$ - in Form von handlungsbezogenen Schleifen, Kreisläufen und Recyclings unter dem Oberbegriff „Loops“ neu zusammengesetzt zu werden: Erst wenn die Menschen sich vergegenwärtigten, dass die Konsequenzen ihres eigenen Tuns - etwa in Form von Verschmutzungen -

98 Vgl. Latour, Anthropocene (Fn. 64).

99 B. Latour, Versuch eines „Kompositionistischen Manifests“, Zeitschrift für Theoretische Soziologie (ZTS) $1 / 2013$, S. 8 ff.

100 Vgl. hierzu und zum Folgenden Latour, Anthropocene (Fn. 64).

101 Sloterdijk, Zeilen (Fn. 69), S. 19 f.

102 Vgl. etwa in diesem Sinn S. Sörlin, Environmental Humanities, BioScience 62 (2012), S. 788: „After half a century of putting nature first, it may be time to put humans first."

103 Vgl. B. Latour, Das Parlament der Dinge, Frankfurt 2010, S. 17, 32, 70, 82, 84, 87, 89, 165, 173, 195, 210 ff., 246, 252, 261, 277, 285; ders., Die Hoffnung der Pandora, Frankfurt 2002, S. 220; ders., Eine neue Soziologie für eine neue Gesellschaft, Frankfurt 2007, S. 22, 73, 205 f., 278, 282, 296, $326 \mathrm{f}$.

104 Latour, Anthropocene (Fn. 64); vgl. auch ders., in: Beck/Latour, Die Apokalypse duldet keinen Sachzwang, FAZ, 15.5.2014, S. 14.

105 Vgl. Latour, Wir sind nie modern gewesen (Fn. 65), S. 114 f., 170; ders., Hoffnung (Fn. 103), S. 219, 372; ders., Parlament (Fn. 103), S. 108, 114, 117 f., 285; ders., Soziologie (Fn. 103), S. 121 ff., 405.

106 Vgl. Latour, Anthropocene (Fn. 64), mit Verweis auf P. Sloterdijk, Sphären II, Frankfurt 1999, S. $465 \mathrm{ff}$. 
zu ihnen selbst zurückkehrten, werde ihnen die so entstehende loopförmige Handlungs- als weitgreifende Verantwortungssphäre überhaupt bewusst. Doch diese Deund Rekomposition erfasst im anthropozänen Kontext nicht nur die Erde als Globus, sondern auch die Erde als Natur: Die Natur existiere nicht als eine vorgegebene Einheit und Ordnung, die wissenschaftlich „nur“ erkannt zu werden brauche. Latour zu Folge leben wir vielmehr in einer postökologischen Epoche, die auf der Suche nach einer Komposition ist, welche die Nachfolge materieller bzw. substanzieller Naturvorstellungen antreten könnte: ein kompositionistisches Konzept aus Akteuren und Aktanten, das Latour - so ver- wie befremdend - nach der mythologischen Erdgöttin Gaia benennt. ${ }^{107}$

Die konkreteren und zugleich weiteren Dimensionen dieses Ansatzes für das politische und daran anschließend für das rechtliche Verständnis des Anthropozän hat Latour in seinem Versuch eines ,Kompositionistischen Manifests' entwickelt, in den viele Einzelströmungen seines bisherigen Werks eingeflossen sind. Auch das Kompositionistische Manifest unterstreicht zunächst, dass „wir durch unseren eigenen Fortschritt und unsere schiere Menge die Erde auf unsere Schultern genommen haben, wie es der bemerkenswerte Neologismus ,Anthropozän` so deutlich zum Ausdruck bringt, dieses neu benannte Erdzeitalter, das mit der industriellen Revolution und deren globalen Folgen einsetzte. “108 Der Kompositionismus akzeptiert für die Konturierung seines Anthropozän-Konzepts jedoch „keine Welt des Dahinter“109 und deshalb auch keine „schon immer dagewesene Natur“, 110 die nur wissenschaftlich erkannt werden müsste und in dieser Erkenntnis zugleich als Referenz für die Kritik kultureller Entwicklung zu Verfügung stünde. ${ }^{111}$ Zwar konstatiert Latour, dass die Unterscheidung zwischen Kultur und Natur die dynamische „Potentialdifferenz" 112 der „Moderne“ gebildet habe. Doch nach dem Kollaps dieser Bifurkation stelle sich die Frage, was nach dem „Ende der Natur“ ${ }^{113}$ komme. Latours Antwort lautet: eine Komposition, also eine Verbindung aus „Immanenz und Wahrheit“, ${ }^{114}$ die „nur durch den langsamen Prozess von Komposition und Kompromiss erreicht werden“ 115 kann. Deshalb ist das Anthropozän für Latour „wieder zu einer Zeit des

107 Vgl. grds. J. Lovelock, Gaias Rache, 2. Aufl., Berlin 2010, S. 29 ff.; zu den Ambivalenzen der GaiaHypothese Latour, Parlament (Fn. 103), S. 13 f., 117, 248, 251, 349; ders., in: Beck/Latour, Apokalypse (Fn. 104), S. 14; krit. zur Gaia-Esoterik Schwägerl, Menschenzeit (Fn. 2), S. 182.

108 Latour, Manifest (Fn. 99), S. 18; ferner ders., Parlament (Fn. 103), S. 76; ders., in: Beck/Latour, Apokalypse (Fn. 104), S. 14.

109 Latour, Manifest (Fn. 99), S. 12.

110 Latour, Manifest (Fn. 99), S. 15.

111 Vgl. Latour, Manifest (Fn. 99), S. 12 ff., 15 ff.

112 Vgl. Latour, Manifest (Fn. 99), S. 15.

113 Latour, Manifest (Fn. 99), S. 13; vgl. auch ders., ebd., S. 18; ders., Parlament (Fn. 103), S. 41.

114 Latour, Manifest (Fn. 99), S. 16 (Hervorhebungen im Original).

115 Latour, Manifest (Fn. 99), S. 16. 
Staunens über die Durcheinander der Natur geworden ". ${ }^{116}$ Auf der Suche nach theoretischen Anknüpfungspunkten für eine Rekomposition dieses Durcheinanders verweist er auf das 16. Jahrhundert, das noch keine vernaturwissenschaftlichte Unterscheidung zwischen handelnden Subjekten und unbelebter Natur gekannt habe. ${ }^{117}$ Die animistische Provokation der Handlungs(un)fähigkeit der unbelebten (Natur-)Objekte und die damit einhergehende Frage ihrer kompositionistischen Reanimation in Form von Aktanten stehen damit im Mittelpunkt von Latours politischer Ökologie. ${ }^{118}$ Diese setzt auf eine langsame und schrittweise Neukomposition kreativer Handlungsfähigkeiten in einer Welt, in welcher sich der Mensch von zwei Vorstellungen verabschieden müsse: einerseits in der Natur gefangen zu sein oder diese einfangen zu können, andererseits in die Natur eingebettet zu sein oder sich selbst in diese einbetten zu können. ${ }^{119}$ Deshalb ist es nach Latour „Zeit zu komponieren - in allen Bedeutungen des Wortes, einschließlich der Konnotationen des Kompromisses, der Vorsicht, der langsamen, behutsamen und vorsichtigen Bewegung." 120 Vorsorge bzw. Prävention wird dabei als „ein wichtiger juristischer Begriff" ${ }^{21}$ identifiziert, den Latour in unmittelbarer Nachbarschaft zu Ulrich Becks Konzeption von Risiko und Risikogesellschaft sieht. ${ }^{122}$ Aufgrund dieser Vorsicht beuge die politische Rekomposition der Welt den beiden großen Fluchtversuchen der „Moderne“ vor, nämlich zum einen alle Innovationen kritisch abzulehnen und zum anderen alle Innovationen unkritisch umzusetzen. ${ }^{123}$ Demgegenüber soll die Politik „zögern, tasten, experimentieren, wieder aufgreifen, immer wieder beginnen, regelmäßig ihre Arbeit an der Komposition auffrischen." 124 Das politische Ziel seines Kompositionistischen Manifests beschreibt Latour abschließend in Reminiszenzen zum Kommunistischen Manifest ${ }^{125}$ von Friedrich Engels und Karl Marx: ${ }^{126}$ Zwischen beiden Manifesten bestehe eine dünne Verbindungslinie „in der Suche nach

116 Latour, Manifest (Fn. 99), S. 19 (Hervorhebung im Original); vgl. auch ders., Wir sind nie modern gewesen (Fn. 65) S. 96; ders., Hoffnung (Fn. 103), S. 34.

117 Vgl. Latour, Manifest (Fn. 99), S. 18 ff., 22.

118 Vgl. Latour, Manifest (Fn. 99), S. 23 f.; vgl. auch ders., Parlament (Fn. 103), S. 123.

119 Vgl. Latour, Manifest (Fn. 99), S. 19 ff.

120 Latour, Manifest (Fn. 99), S. 26; ferner auch ders., Parlament (Fn. 103), S. 223 ff.; ders., Soziologie (Fn. 103), S. 286 ff., bes. S. 295 f.

121 Latour, Manifest (Fn. 99), S. 26 Fn. 47.

122 Vgl. Latour, Manifest (Fn. 99), S. 26 Fn. 47; ferner auch ders., Parlament (Fn. 103), S. 37, 315, 332; ders., Soziologie (Fn. 103), S. 139 f., 324; ders., Krieg der Welten - wie wäre es mit Frieden?, Berlin 2004, S. $47 \mathrm{f}$.

123 Vgl. Latour, Manifest (Fn. 99), S. 26.

124 Latour, Manifest (Fn. 99), S. 29.

125 Vgl. K. Marx/F. Engels, Manifest der Kommunistischen Partei, in: Marx, Die Frühschriften, Stuttgart 1971, S. $525 \mathrm{ff}$.

126 Vgl. hierzu und zum Folgenden Latour, Manifest (Fn. 99), S. 27; ferner ders., Soziologie (Fn. 103), S. 241, $324 \mathrm{ff}$. 
dem Gemeinsamen“. ${ }^{127}$ Diese „Sehnsucht nach der Gemeinsamen Welt“"128 sei vom Kommunismus in den Kompositionismus eingegangen - allerdings mit dem Unterschied, dass die Welt langsam komponiert werden müsse, anstatt sie als gegeben anzusehen und allen aufzuzwingen. Die Menschheit gerate wieder in Bewegung, vertrieben aus der Utopie der Ökonomie, die zur Dystopie geworden sei, und auf der Suche nach einer Utopie der Ökologie, von der niemand wisse, wie man sie erfüllen könne. Deshalb laute die einzig relevante Frage des Kompositionistischen Manifests im Anthropozän: „Wie kann eine lebenswerte und atembare ,Heimstatt' für diese fehlgeleiteten Massen aufgebaut werden?" 129 Wenn es für die „Menschheit“ „keinen dauerhaften Platz auf Pandora gibt, wie werden wir eine nachhaltige Heimat auf Gaia finden? “ 130

\section{Konzeptkritik}

Zweifellos zählt das Kompositionistische Manifest gegenwärtig zu den innovativsten Ansätzen für ein neues Gesellschaftsverständnis „zwischen“ Realismus und Konstruktivismus. Der Kompositionismus eröffnet nicht nur ungewöhnliche und überraschende Perspektiven, sondern ist weit über die Geistes- und Sozialwissenschaften hinaus interdisziplinär anschlussfähig. Zugleich zeigen sich jedoch im interdisziplinären Dialog auch die aktuellen Grenzen des kompositionistischen Theorieangebots im Allgemeinen und des kompositionistischen Anthropozän-Konzepts im Besonderen. Mit der gezielten Dekonstruktion von überkommenen Differenzierungen - wie beispielweise zwischen Kultur und Natur oder Wissenschaft und Wahrheit - geht ein wissenschaftlicher Kriterien- und sozialer Orientierungsverlust einher, der durch eine Re- bzw. Neukomposition ordnungsstiftender Leitbegriffe kompensiert werden müsste, aber derzeit (noch) nicht (vollständig) kompensiert wird.

\section{1. „Rechtsstaat der Natur“}

Der Kompositionismus reflektiert mit seiner Dekonstruktion der überkommenen ordnungsstiftenden Einheitsbegriffe - Kultur, Natur, Öffentlichkeit, Person, Politik, Recht, Staat, Wahrheit und Wissenschaft - eine emotionale wie kognitive Grunderfahrung, welche die individuelle und kollektive Erlebniswelt in den letzten drei Jahrzehnten geprägt hat. Dies führt jedoch zu einem Verlust von ordnungsstiftenden Unterscheidungen - wie beispielsweise zwischen Kultur und Natur - oder entgrenzt funktionale Differenzierungen - wie etwa zwischen Politik und Ökonomie. Mit diesen Entgrenzungen und Entdifferenzierungen gehen individuelle und kollektive Orientierungsverluste einher: Was tritt an die Stelle der Unterscheidung von Kultur und Natur oder an die Stelle der Grenzziehung zwischen Politik und Ökonomie? Der

127 Latour, Manifest (Fn. 99), S. 27.

128 Latour, Manifest (Fn. 99), S. 27.

129 Latour, Manifest (Fn. 99), S. 27.

130 Latour, Manifest (Fn. 99), S. 27. 
Kompositionismus kann hier eine inhaltliche und eine modale Rekomposition anbieten. Beide Ansätze finden sich auch im kompositionistischen Anthropozän-Konzept. So dekomponiert Latour die einheitliche Vorstellung des Globus, um die Rundheit der Erde als Multitude von Handlungs- und Verantwortungssphären zu rekomponieren. Demgegenüber bietet er jedoch für die De- und Rekomposition der Differenzierung zwischen Kultur und Natur, die für das Verständnis des Anthropozän von zentraler Bedeutung ist, „nur“ strukturelle Hinweise an, wie die Rekomposition erfolgen kann. Auf die Frage „Was folgt auf die Natur?"131 lautet die Antwort: eine „Komposition“, über deren langsames, tastendes und vorsichtiges Entstehen das Kompositionistische Manifest lediglich modale Andeutungen enthält, ohne materielle Kriterien für das Verhältnis von natürlichen und kulturellen Komponenten in diesem neuen Kompositum zu entwickeln.

Dies wird noch deutlicher, wenn man das Kompositionistische Manifest vor dem Hintergrund des Konzepts des „Rechtsstaats der Natur" ${ }^{132}$ liest, das Latour in Das Parlament der Dinge konturiert hat: ${ }^{133}$ Die Welt entfaltet sich in vernetzten Beziehungen von menschlichen Akteuren und nichtmenschlichen Aktanten, für deren hybriden Charakter Latour beispielhaft auf das Ozonloch und den Klimawandel verweist: ${ }^{134}$ Beide sind durch menschlichen Einfluss mit entstanden, bestimmen aber auch wiederum selbst menschliches Handeln mit. In dieser hybriden Welt „bedarf es dennoch einer Repräsentation der Assoziationen zwischen Menschen und nichtmenschlichen Wesen durch ein explizites Verfahren, damit sich entscheiden läßt, wodurch sie in einer künftigen gemeinsamen Welt versammelt und vereint werden.“135 Für die „Verfassung"136 dieses Kollektivs schlägt Latour die Bildung eines Oberhauses und eines Unterhauses vor: Das Oberhaus stellt die Freund-Feind-Frage ${ }^{137}$ und entscheidet damit, wer Mitglied des politischen Akteurs- und AktantenKollektivs ist. ${ }^{138}$ Im Anschluss daran entscheidet das Unterhaus über die Konflikte der versammelten Akteure und Aktanten, indem es deren Interessen in eine hierar-

131 Latour, Manifest (Fn. 99), S. 15.

132 Latour, Parlament (Fn. 103), S. 225, 256, 279.

133 Vgl. hierzu und zum Folgenden Latour, Parlament (Fn. 103), S. 172 ff., bes. S. 210 ff.

134 Vgl. auch Latour, Wir sind nie modern gewesen (Fn. 65) S. 7 ff., 18 ff., 22 ff., 43 ff., 49, 55 ff., 67 ff.; ferner ders., Hoffnung (Fn. 103), S. 27, 111 f., 247 f.; ders., Soziologie (Fn. 103), S. 95, 102 ff., 117, $121 \mathrm{ff} ., 405$.

135 Latour, Parlament (Fn. 103), S. 59; vgl. ders., ebd., S. 58, 285; ders., Wir sind nie modern gewesen (Fn. 65), S. 11, 120, 183, 191; ders., Hoffnung (Fn. 103), S. 211 ff.; ders., Soziologie (Fn. 103), S. 17 ff., 22 f., 121 ff., 129, 205 f., 223, 278, 282, 296, 424 ff., 436, 440, 443 ff.

136 Vgl. für die metaphorische Verwendung des Verfassungsbegriffs Latour, Parlament (Fn. 103), S. 211; ders., Wir sind nie modern gewesen (Fn. 65), S. 24; ders., Soziologie (Fn. 103), S. 436.

137 Vgl. Latour, Parlament (Fn. 103), S. 190, 225 ff., 261 f., 288; ferner ders., ebd., S. 227, 346, 350, und ders., in: Beck/Latour, Apokalypse (Fn. 104), S. 14, zur Rezeption von Carl Schmitts „Begriff des Politischen“ - vgl. C. Schmitt, Der Begriff des Politischen, 3. Aufl., Berlin 1963 (1991), S. 26 f.

138 Vgl. Latour, Parlament (Fn. 103), S. 211 ff., 229. 
chische Ordnung bringt. ${ }^{139}$ Das Problem dieser neuen Verfassung des „Rechtsstaats der Natur" liegt nicht so sehr in der etwas unglücklichen Rezeption von Carl Schmitts Der Begriff des Politischen: Der Feind bei Schmitt ist die Person, die schlicht existenziell ausgelöscht werden kann. ${ }^{140} \mathrm{Im}$ Gegensatz dazu besteht Latour darauf, dass die vom Oberhaus abgelehnten Akteure und Aktanten ein Berufungsrecht im Hinblick auf ihre Aufnahme haben und in kommenden Konstellationen auch aufgenommen werden müssen. ${ }^{141}$ Vielmehr bleibt in der juristischen Perspektive offen, nach welchen Kriterien das Oberhaus Akteure und Aktanten akzeptiert und das Unterhaus deren Interessen bewertet. Eine bloße Abbildung faktischer Hybrid-Konstellationen im ersten Fall und eine schlichte Widerspiegelung faktisch gegebener Interessenrankings im zweiten Fall genügen juristisch gerade nicht. Doch normative Kriterien, welche diese Inklusions- und Abwägungsprozesse steuern könnten, finden sich nicht in der Verfassung des „Rechtsstaats der Natur“. Dies hängt auch mit dem Verständnis von Normativität zusammen, das Latour in seiner politischen Ökologie verfolgt: Die abstrakte Unterscheidung zwischen Sein und Sollen wird dekonstruiert ${ }^{142}$ und sodann in der Vorstellung rekomponiert, dass sich Legitimation und Normativität erst in den Verfahren generieren, welche die Verfassung des „Rechtsstaats der Natur“ vom Oberhaus zum Unterhaus vorzeichnen. ${ }^{143}$ In dieser kompositionistischen Variation einer Legitimation durch Verfahren genießen materiell vorgefasste Entscheidungskriterien im Hinblick auf die Akzeptanz von Akteuren bzw. Aktanten durch das Oberhaus sowie für die Konfliktabwägungen des Unterhauses aber offensichtlich keinen normativen Stellenwert. Die Verfassung des „Rechtsstaats der Natur“ bleibt rein modal und strukturell formal.

Die Orientierungsverluste, welche mit diesem Konzept einer „reinen“ Komposition einhergehen, zeigen sich auch im zentralen anthropozänen Streitfall um den Klimawandel, den Latour im Kompositionistischen Manifest mit Verweis auf das „Kopenhagener Klimagate" verhandelt: ${ }^{144}$ Zweifler am Phänomen des anthropogen verursachten Klimawandels wiesen kritisch - so Latour - auf dessen Konstruktion im Spannungsfeld von Natur, Politik, Wissenschaft und Wahrheit hin. Für Latour trägt dieser kompositionistische Einwand gegen die Diagnose des anthropogenen Klimawandels jedoch nicht: Vielmehr veranschauliche er nur den Unterschied zwischen einer guten und einer schlechten Komposition. Doch welche Kriterien für eine gute oder schlechte Komposition entscheidend sind, wird nicht offengelegt. Latours Kon-

139 Vgl. Latour, Parlament (Fn. 103), S. 211, 220 ff., 229 f., 256.

140 Vgl. Schmitt, Begriff (Fn. 137), S. 33, 46, 49, 70.

141 Vgl. Latour, Parlament (Fn. 103), S. 166, 187, 201 f., 207, 227, 235, 246, 249 f., 288.

142 Latour, Parlament (Fn. 103), S. 167, 216, 237, 280 f.

143 Vgl. Latour, Parlament (Fn. 103), S. 59, 127, 165, 167, 216, 222, 227 f., 235, 244, 251 ff., 279, 295 f., 330 f.; ferner ders., Soziologie (Fn. 103), S. 277, 438 f.

144 Vgl. hierzu und zum Folgenden Latour, Manifest (Fn. 99), S. 15 f.; ferner ders., in: Beck/Latour, Apokalypse (Fn. 104), S. 14. 
zeption für Eine neue Soziologie für eine neue Gesellschaft lässt sich zwar entnehmen, dass ein Akteur-Netzwerk-Bericht dann gelungen ist, wenn er die jeweilige AkteursAktanten-Konstellation hinreichend komplex entfaltet. ${ }^{145}$ Aufgrund welcher Kriterien - so muss man Latour fragen - ist dies aber in der politischen Ökologie der Fall? Warum war „Kyoto“ seiner Auffassung nach ein Erfolg146 und „Kopenhagen“ ein Flop? Latour „sperrt“ für seine Akteur-Netzwerk-Theorie die ganz zentrale Funktion des Wahrheitsbegriffs, nämlich die Freiheit der Wissenschaft gegen politische Kompromisse rechtlich zu immunisieren. Deshalb bleiben ihm letztlich nur zwei Möglichkeiten: Er kann einerseits die wissenschaftspolitischen Verhandlungsergebnisse der Klimagipfel nur so akzeptieren, wie sie sind. Oder er muss andererseits wissenschaftliche und politische Kriterien wenn nicht neu komponieren, so doch jedenfalls offen benennen, anhand derer sich die hinreichende Komplexität für das Gelingen oder Scheitern einer Komposition bestimmt. Doch das Konzept der „reinen “ Komposition lässt diese materielle Kriterienfrage (bewusst) offen. ${ }^{147}$

\section{Nichtreflexive Loops}

Das Fehlen von materiellen Kriterien für eine Neukomposition des Verhältnisses von Kultur und Natur schlägt sich auch in der anthropozänen Konzeption der Erde als Multitude reflexiver Handlungs- und Verantwortungssphären nieder. Durch die Reflexivität von Handlungsschleifen als Loops soll den Akteuren ihre Verantwortung bewusst werden. Doch in der anthropozänen Realität stellt diese geschlossene Reflexivität von Handlungs(folgen)kreisläufen gerade nicht den Regelfall dar. Die Handlungen von Akteuren wirken regelmäßig nicht auf die eigene Lebensführung zurück, sondern betreffen andere Akteure an anderen Orten und zu anderen Zeiten. ${ }^{148}$ Abfall wird zum Beispiel vergraben, verschoben oder schlicht „vergessen“, sodass er in der Lebenswelt anderer oder künftiger Menschen spürbar wird. ${ }^{149}$ Konsum führt zu Artensterben, dessen Folgen sich aber gerade nicht im Alltag der in jeder Hinsicht optimal geschützten Verbraucher der OECD-Welt, sondern wiederum für „ferne“ Menschen oder künftige Generationen realisieren. ${ }^{150}$ Selbst hinsichtlich des Klimawandels, auf den die Komposition dieses loopförmigen Verantwortungsrecyclings passgenau zugeschnitten erscheint, unterscheiden sich Verursacher, die gegenwärtig in der OECD-Welt leben, und die unmittelbar Betroffenen im Globalen Süden sowie wiederum künftige Generationen - und dies ganz unabhängig davon, dass sich auch manche als Gewinner des globalen Klimawandels fühlen mögen. ${ }^{151}$ In der in-

145 Vgl. Latour, Soziologie (Fn. 103), S. 210, 211 ff., 224 ff., 270.

146 Vgl. Latour, Parlament (Fn. 103), S. 85 f., 96, 146.

147 Vgl. Latour, Parlament (Fn. 103), S. 165.

148 Vgl. U. Beck, Die Neuvermessung der Ungleichheit unter den Menschen, Frankfurt 2008, S. 29 ff., bes. S. 31.

149 Vgl. T. H. Eriksen, Mensch und Müll, Basel 2013, S. 41 ff., 81 ff., 233 ff.

150 Vgl. Kersten, Dickicht (Fn. 70), S. 15 ff.

151 Vgl. Sloterdijk, Zeilen (Fn. 69), S. 472 f., zum „Norwegeneffekt“. 
haltlich als Handlungs- und Verantwortungssphären rekonstruierten Welt sind also die reflexiv komponierten Loops keineswegs geschlossen. Es sei denn, man führte den Kollektivsingular „Menschheit“ wieder ein, auf den Abfall, Artensterben und Klimawandel zurückwirken könnten. Doch die politische Konstitution eines „Anthropos" liegt grundsätzlich jenseits des kompositionistischen Ansatzes. Aufgrund der fehlenden Reflexivität der Loops bedarf es deshalb anderer Kriterien für die Zurechnung von anthropozänen Handlungs- und Verantwortungssphären. Die schlichte Erhöhung der Zahl der Akteure durch die Anerkennung nichtmenschlicher Aktanten hilft hier nicht weiter. Nur in einem System reflexiv geschlossener Handlungsund Verantwortungsloops kann eine rein numerische Steigerung der Akteure und Aktanten zu einer anthropozänen Sphärenverdichtung führen. In einem durch die Realität aufgebrochenen System der Handlungs- und Verantwortungssphären steigert jedoch die Ausweitung von Agency nur das Zurechnungsproblem von Handlungsfolgen. ${ }^{152}$ Das somit notwendige Zurechnungskonzept lässt sich allein durch eine materielle Neubestimmung des Verhältnisses von Kultur und Natur entwickeln. Dieses Zurechnungskonzept könnte naturwissenschaftliche Wirkungszusammenhänge und kulturelle Normvorstellungen in einem neuen Verständnis von Umweltgerechtigkeit rekomponieren. ${ }^{153}$ In diese iterative Rekomposition von Environmental Justice würden auch die aktuellen wie historischen Verantwortungen für Artensterben, Klimawandel und Ressourcenverschwendung einfließen. Doch an Stelle dieser sicherlich komplexen Agenda setzt das Kompositionistische Manifest vor allem auf das Vorsorgeprinzip.

\section{Risikovorsorge}

Das kompositionistische Anthropozän-Konzept favorisiert eine behutsame, kompromisshafte, langsame, schrittweise und vorsichtige Politik: „Wir erkennen, “ - so Latour - „dass wir ein zivilisiertes Gemeinwesen nur schrittweise bauen können. Und auf diese Weise nach einem neuen Leviathan suchen, der mit Gaia fertig wird. “154 Diese Suche ist Ausdruck eines neuen „Lernvertrags“, 155 der an die Stelle des alten Sozialvertrags treten und „die gemeinsame Unwissenheit von Regierenden und Regierten in der Situation des kollektiven Experimentierens " 156 zum Ausdruck bringen soll. Der Lernvertrag ist grundsätzlich dem Vorsorgeprinzip verpflichtet, das $L a$ tour im Kontext von Ulrich Becks Konzept des Risikos und der Risikogesellschaft

152 Vgl. Latour, Soziologie (Fn. 103), S. 79, zum Konzept von Agency.

153 Vgl. demgegenüber zur derzeitigen Kontur von Umweltgerechtigkeit bzw. Environmental Justice zwischen politischer Bewegung, umweltbezogenem Raumkonzept und globalen Gerechtigkeitsperzeptionen M. Kloepfer, Environmental Justice und geographische Umweltgerechtigkeit, DVBl. 2000, S. 750 ff.; ders., Umweltgerechtigkeit, Berlin 2006.

154 Latour, in: Beck/Latour, Apokalypse (Fn. 104), S. 14.

155 Latour, Parlament (Fn. 103), S. 260, 292.

156 Latour, Parlament (Fn. 103), S. 292; vgl. auch ders., ebd., S. 260. 
verortet. ${ }^{157}$ Risikogesellschaften entstehen, wenn das Eingehen von Risiken nicht mehr sozial kompensiert werden kann, beispielsweise durch eine Versicherung. In diesem Fall werden Risiken gesamtgesellschaftlich riskant, was Beck auf die einprägsame Formel von der „neue[n] Riskantheit des Risikos“158 gebracht hat. Dies motiviert Gesellschaften, die Präventionsschwelle vorzuverlegen, indem sie Risikovorsorge betreiben. Aber auch die Risikogesellschaft kennt ihre Aporien, die sich in zwei gegenläufigen Tendenzen niederschlagen. ${ }^{159}$ Auf der einen Seite überspielt vor allem die allgegenwärtige öffentliche Rede von der „Risikogesellschaft“ die Gefahrenwahrnehmung: Die Bürgerinnen und Bürger halten Gefahren für Risiken, die über die Kombination von technischer Vorsorge und praktisch ausgeschlossenem Restrisiko sozial akzeptabel erscheinen. Das paradigmatische Beispiel hierfür ist die Atomkraft. Auf der anderen Seite hat die Risikogesellschaft Regulierungsreflexe ausgelöst, die je nach kultureller Risikoperzeption zu einem faktischen Ausschluss der Wahrnehmung von riskanten Freiheitsrechten führen. ${ }^{160}$ Als paradigmatisches Beispiel hierfür kann - jedenfalls in der Bundesrepublik - die Regulierung der Grünen Gentechnik gelten. ${ }^{161}$ Damit changiert die Risikogesellschaft zwischen defensiver Gefahrenignoranz einerseits und der überschießenden Bewertung von Worst-Case-Szenarien andererseits. ${ }^{162}$ Deshalb ist eine holistische Risikosoziologie für die Sicherheit und die Freiheit liberaler Gesellschaften letztlich selbst zu risikoreich. Schon diese grundsätzlichen Einwände sprechen gegen die theoretische Stilisierung der Risikovorsorge zum einzigen und alles bestimmenden gesamtgesellschaftlichen Paradigma, also gegen die „Risikogesellschaft“. Sie stellen aber insbesondere die Rezeption eines allumfassenden Vorsorgeprinzips durch ein kompositionistisches AnthropozänKonzept in Frage, das sich schon methodologisch einen Pluralismus von Kompositionsprinzipien leisten könnte, wenn nicht sogar leisten müsste.

Allerdings hat Beck in den letzten Jahren seine Theorie der Risikogesellschaft vor allem in Bezug auf den Klimawandel zu einer kosmopolitischen Theorie der Weltrisikogesellschaft fortgeschrieben: ${ }^{163}$ Der Klimawandel setze ein „kosmopolitisches Moment ${ }^{\star 164}$ frei. Er verschmelze zum einen soziale und natürliche Ungleichheit ${ }^{165}$

157 Vgl. hierzu und zum Folgenden U. Beck, Risikogesellschaft, Frankfurt 1986, S. 7 ff., 25 ff., 67 ff., 300 ff.; ders., Weltrisikogesellschaft, Frankfurt 2007, S. 19 ff., 24 ff.; ders., Neuvermessung (Fn. 148), S. 55 f.; ders., in: ders./Latour, Apokalypse (Fn. 104), S. 14.

158 Beck, Weltrisikogesellschaft (Fn. 157), S. 24 (Klammerzusatz durch den Verfasser).

159 Vgl. hierzu und zum Folgenden aus verfassungsrechtlicher Perspektive J. Kersten/A. Ingold, Die Beschleunigung des Atomausstiegs, ZG 2011, S. 350 (359 ff.).

160 Vgl. C. Sunstein, Gesetze der Angst, Frankfurt 2007, S. 25 ff.

161 Vgl. H. Grimm/S. Schleissing (Hrsg.), Grüne Gentechnik, Baden-Baden 2012.

162 Vgl. Stunstein, Gesetze (Fn. 160), S. 97 ff.; ders., Worst-Case-Scenarios, Cambridge/London 2007, S. $1 \mathrm{ff}$.

163 Vgl. Beck, Weltrisikogesellschaft (Fn. 157), S. 94 ff.

164 Beck, Weltrisikogesellschaft (Fn. 157), S. 109 ff.; ders., Neuvermessung (Fn. 148), S. 39.

165 Vgl. Beck, Neuvermessung (Fn. 148), S. 25. 
und wirke zum anderen hierarchisch und demokratisch, weil ihm niemand entgehe. ${ }^{166}$ Aus diesem Grund kosmopolitisiere sich der menschliche Alltag: ${ }^{167}$ Angesichts der globalen Risiken würde jedermann mit den eigenen Handlungsfolgen für andere, scheinbar ferne Menschen und künftige Generationen konfrontiert. Hieraus ergibt sich für Beck der „kosmopolitische Imperativ: Grenzübergreifende Lebens- und Überlebenslagen lassen sich nur in einer kosmopolitischen Perspektive - das heißt neu zu findenden, neu zu definierenden, grenzüberschreitenden Fokussen, die die national ausgeschlossenen Anderen einbeziehen - deutend verstehen und ursächlich erklären. "168 Auf den ersten Blick scheint diese theoretische Adaption der Risikogesellschaft an einen sozialpolitisierten und verfassungsbegrifflich aufgeladenen Klimawandel in der Lage zu sein, die kompositionistische Lücke zu schließen, welche die fehlende Reflexivität der loopbasierten Handlungs- und Verantwortungssphären hinterlassen hat: Die kosmopolitische Wende führt jedem Menschen die eigenen Handlungsfolgen für andere vor Augen und auf diese Weise zu einem kosmopolitischen Verantwortungsbewusstsein. Aber jenseits dieser ersten Evidenz sind die kosmopolitische Neuauflage der Risikogesellschaft und das kompositionistische Anthropozän-Konzept nicht anschlussfähig: ${ }^{169} \mathrm{Zwar}$ verabschieden beide grundsätzlich den Dualismus von Kultur und Natur. ${ }^{170}$ Sie geben jedoch unterschiedliche Antworten auf die Frage: „Wie gehen wir mit der Natur nach ihrem Ende um?" 171 Beck hat seine Vorstellung von der „,Vergesellschaftung“ der Natur”172 in der Risikogesellschaft ${ }^{173}$ zu einem „institutionellen Konstruktivismus “ 174 der Weltrisikogesellschaft weiterentwickelt, in der „Natur“ industriell verinnerlicht und institutionell definiert und produziert wird. ${ }^{175}$ Demgegenüber löst Latour den Dualismus von Kultur und Natur nicht einseitig zur Gesellschaft hin auf, sondern konturiert ein neues, postnatürliches Kompositum. Die dafür notwendige, kompositionistische Politik lässt sich jedoch nicht durch Risiko und Vorsorge konzeptionalisieren. Sicherlich weist $\mathrm{La}$ tour in seiner Konzeption des „Rechtsstaats der Natur" durch die Inklusionsentscheidung des Oberhauses Akteure und Aktanten aus, deren Güter in den Konfliktabwägungen des Unterhauses im Hinblick auf Risiken bewertet werden könnten. Doch um eine Risikoabwägung vornehmen zu können, bedarf es nicht nur der Anerkennung der risikobegründenden und risikobetroffenen Akteure bzw. Aktanten

166 Vgl. Beck, Neuvermessung (Fn. 148), S. 39.

167 Vgl. hierzu und zum Folgenden Beck, Neuvermessung (Fn. 148), S. 31, 40 f., 50 f.; ders., Weltrisikogesellschaft (Fn. 157), S. $118 \mathrm{ff} ., 170 \mathrm{ff}$.

168 Beck, Neuvermessung (Fn. 148), S. 49.

169 Vgl. hierzu und zum Folgenden auch Beck/Latour, Apokalypse (Fn. 104), S. 14.

170 Vgl. Beck, Weltrisikogesellschaft (Fn. 157), S. 170; Heise, Natur (Fn. 70), S. 14 f.

171 Beck, Weltrisikogesellschaft (Fn. 157), S. 170 (Hervorhebung im Original).

172 Heise, Natur (Fn. 70), S. 15.

173 Vgl. Beck, Risikogesellschaft (Fn. 157), S. $107 \mathrm{ff}$.

174 Beck, Weltrisikogesellschaft (Fn. 157), S. 170.

175 Vgl. Beck, Weltrisikogesellschaft (Fn. 157), S. 170 ff. 
sowie Güter, sondern vor allem auch der Risikozurechnung und Risikogewichtung. Die materiellen Kriterien, welche diese Zurechnungs- und Abwägungsentscheidungen überhaupt erst ermöglichen, werden jedoch (bisher) von der Verfassung des „Rechtsstaats der Natur“ (noch) nicht generiert. Jenseits der grundsätzlichen Einwände gegen die Theorie der Risikogesellschaft fehlt es damit dem kompositionistischen Anthropozän-Konzept auch an der Möglichkeit, das von ihm favorisierte Prinzip der Risikovorsorge unmittelbar anzuwenden.

\section{Konzeptdimensionen}

Die Einordnung der kompositionistischen Sphärenvorsorge als Anthropozän-Konzept muss, an dessen vier Dimensionen - Zeit, Raum, Akteur und Wissenschaft gemessen, ambivalent ausfallen. Im Grundsatz erweist sich das kompositionistische Anthropozän-Konzept in jeder Hinsicht als entwicklungsoffen: Es fokussiert in der zeitlichen Dimension nicht auf eine Zukunft, sondern komponiert eine Vielzahl künftiger Perspektiven. Es introvertiert sich nicht in der klaustrophoben Vorstellung des einen Planeten, sondern betont die Multitude von Handlungs- und Verantwortungssphären (Loops). Es fixiert nicht auf die Menschheit als neuen politischen Körper (Anthropos) des Anthropozän, sondern auf die Vielheit von menschlichen Akteuren und nichtmenschlichen Aktanten. Schließlich versteht es die Wissenschaft als eine Anleitung zu kompositionistischer Arbeit und Politik. ${ }^{176}$ Doch eine Kritik von Latours Konzeptualisierung des Anthropozän kommt nicht umhin, auf dessen Kriterienarmut zu verweisen. Das Kompositionistische Manifest lässt sich ganz bewusst nur auf eine modale Strukturierung des postnatürlichen Kompositums ein. Damit fehlt es an den materiellen Kriterien, mit denen sich die zeitlichen Perspektiven, die räumlichen Verantwortungssphären, die Vielzahl der Akteure und Aktanten sowie gute, schlechte und scheiternde wissenschaftliche und politische Komposition bewerten ließen. Da der Verweis auf das Konzept der Risikovorsorge in kompositionistischer Perspektive nicht verfangen kann, vermag Latour sich auch nur mit einer idealistischen Übersprungshandlung über die materielle Kriterienarmut seiner „reinen" Komposition hinwegzusetzen, indem er eine Parallele zum Kommunistischen Manifest zieht: ${ }^{177}$ In der "Suche nach dem Gemeinsamen“ werden ideologisch aufgeladene Einheitsbegriffe und Großformeln, welche die politischen Diskurse vom 19. bis zum 21. Jahrhundert geprägt haben, vollkommen unvermittelt im letzten Absatz des Kompositionistischen Manifests zusammenkomponiert: Atmen, Heimat, Klima, Masse, Menschheit, Nachhaltigkeit, Wissenschaft und - nicht zu vergessen - Gaia. Doch der Kompositionismus ist nicht die erste Theorie, die mit dem Vorsatz einer Entzauberung der Welt begonnen hat, um mit einer Neubezauberung der Welt zu enden.

176 Vgl. Latour, Parlament (Fn. 103), S. 180 ff., 300.

177 Vgl. oben C.I. 


\section{Konflikt}

In Abgrenzung zum kontraktualistischen und kompositionistischen Verständnis wird im Folgenden ein konfliktbezogenes Anthropozän-Konzept befürwortet, das sich im Hinblick auf anthropozäne Konfliktkultur, Konflikttypen und Konfliktakteure skizzieren lässt.

\section{Konfliktkultur}

Das Anthropozän war in der Vergangenheit, ist in der Gegenwart und wird in der Zukunft ein Erdzeitalter der lokalen, regionalen und globalen Konflikte sein. Vor allem wohlfahrtsökonomische Interessen an Energie und Ressourcen treiben diese Konflikte an. Es gilt hier nach wie vor die von Werner Sombart überlieferte Einschätzung Max Webers: „Als ich einmal mit Max Weber über die Zukunftsaussichten sprach und wir die Frage aufwarfen: wann wohl der Hexensabbat ein Ende nehmen würde, den die Menschheit in den kapitalistischen Ländern seit dem Beginne des 19. Jahrhunderts aufführt, antworte er: ,Wenn die letzte Tonne Erz mit der letzten Tonne Kohle verhüttet sein wird.“"178 Oder wie Weber auch in Die protestantische Ethik und der Geist des Kapitalismus schreibt: „bis der letzte Zentner fossilen Brennstoffs verglüht ist. " 179 Bisher gehen Artensterben, Klimawandel, Landschaftstransformationen, Müllakkumulation und Umweltverschmutzung mit der dynamischen Entwicklung der sich globalisierenden Wettbewerbs- und Wohlstandsökonomien einher. Doch die Konfliktlinien verlaufen nicht nur abstrakt zwischen Ökonomie und Ökologie, sondern zugleich ganz konkret zwischen Kulturen und Naturen, zwischen Menschen in ihren vielgestaltigen sozialen, ökologischen, politischen und wirtschaftlichen Kontexten und Systemen sowie zwischen der jeweiligen Vergangenheit, Gegenwart und Zukunft. ${ }^{180}$ Es ist gefährlich, diese asymmetrischen und asynchronen Konflikte durch postnatürliche Gestaltungskonzepte zu überspielen. Denn in den gesellschaftlichen Gestaltungsvisionen, die das „Ende der Natur“ begleiten, drohen ökologische Interessen ihre (verbliebene) Widerständigkeit (vollkommen) einzubüßen. Schon Theodor W. Adorno hat diese Gefahr beschrieben, die eine kulturelle Vereinnahmung der Natur mit sich bringt: „Die Rationalisierung der Kultur, welche der Natur die Fenster aufmacht, saugt sie dadurch vollends auf und beseitigt mit der Differenz auch das Prinzip von Kultur, die Möglichkeit zur Versöhnung. "181 Wird aber die Natur von der Kultur aufgesogen, geht auch der Naturschutz nach der Auffassung Adornos letztlich an der Natur vorbei: „Der Naturschutzpark rettet nicht die Natur und stellt sich über kurz oder lang im gesellschaft-

178 W. Sombart, Der moderne Kapitalismus, III/2, Berlin 1928, S. 1010.

179 M. Weber, Die protestantische Ethik und der Geist des Kapitalismus, in: ders., Gesammelte Aufsätze zur Religionssoziologie I, 9. Aufl., Tübingen 1988, S. 17 (203).

180 Vgl. auch Latour, in: Beck/Latour, Apokalypse (Fn. 104), S. 14.

181 T. W. Adorno, Minima Moralia, in: ders., Gesammelte Schriften, 4. Bd., Frankfurt 1997, S. 132. 
lichen Getriebe bloß als Verkehrshindernis heraus. “182 Mit dem Verlust ihrer Widerständigkeit kann also die Natur wohlfahrtsökonomisch umso einfacher konsumiert werden. Auch der sozial wie wirtschaftlich nachgiebige Nachhaltigkeitsgrundsatz wird daran nichts ändern. Damit soll nicht gesagt sein, dass ökonomische Interessen prinzipiell „schlecht“ und ökologische Belange grundsätzlich „gut“ seien. Wohl aber ist gemeint, dass in einer anthropozänen Konfliktkultur die Konflikte zwischen Ökonomie und Ökologie fairer, härter und transparenter ausgetragen werden können und auch müssen, als dies in einer von vornherein auf Ausgleich, Balance, Harmonie oder Symbiose fokussierenden Nachhaltigkeitskultur der Fall wäre. Es kommt - in kompositionistischer Diktion gesprochen - also darauf an, nach dem „Ende der Natur“ als kultureller Vorstellung einer natürlichen Ordnung, die „Widerständigkeit von Natur“ in einer kontroversen Konfliktkultur auszudifferenzieren. Deshalb unterscheidet die anthropozäne Konfliktkultur nicht nur weiterhin zwischen Kultur und Natur. Vielmehr will sie die ganze Komplexität der Konflikte zwischen Kultur und Natur, zwischen Ökonomie und Ökologie, zwischen gegenwärtigen und künftigen Generationen aufgreifen, benennen, publizieren und - natürlich - von Einzelfall zu Einzelfall auch „lösen“. Eine solche anthropozäne Konfliktkultur steigert ganz bewusst die Komplexität von Konflikten, was sich in einer gezielten Steigerung von anthropozänen Konflikttypen und Konfliktakteuren niederschlägt.

\section{Konflikttypen}

Wenn es einer anthropozänen Konfliktkultur um die Steigerung der Konfliktkomplexität gehen soll, kann sie sich nicht nur auf eine Kategorie zur Konfliktlösung festlegen. Dies unterscheidet das Konflikt- vom Kontrakt- und vom Kompositionsmodell, die auf den Nachhaltigkeitsgrundsatz bzw. das Vorsorgeprinzip fokussieren. Dem konfliktorientierten Anthropozän-Konzept genügt die Nachhaltigkeit oder die Risikovorsorge jeweils für sich genommen jedoch aus zwei Gründen nicht: Zum einen führt die Identifikation ganzer Gesellschaftsmodelle mit den Kategorien der Nachhaltigkeit oder des Risikos zum Verlust von Problembewusstsein und zu Gefährdungen von Freiheit und Sicherheit. Zum anderen bildet die normative Fokussierung auf nur ein Prinzip die Konflikte, die das Anthropozän prägen, nicht in angemessener Komplexität ab. Das anthropozäne Konfliktpotenzial kann dabei sowohl aus ökologischer als auch aus kultureller Perspektive spezifiziert werden.

Aus ökologischer Perspektive lässt sich dazu an das Konzept von Ökosystemen anknüpfen: Ökosysteme sind wissenschaftliche Konstruktionen der Natur, die aufgrund einer Wechselwirkung von Organismen mit ihrer unmittelbaren Umwelt als

182 T. W. Adorno, Individuum und Organisation, in: ders., Schriften (Fn. 181), 8. Bd., S. 440 (454); vgl. auch M. Horkheimer/T. W. Adorno, Dialektik der Aufklärung, ebd., 3. Bd., S. 289. 
Einheit aufgefasst werden. ${ }^{183}$ Insbesondere Josef Reichholf hat darauf hingewiesen, dass die Konstruktion von Ökosystemen als Einheit aber nicht dazu führen darf, diese mit Gleichgewichtszuständen zu identifizieren. Ökosysteme verändern sich fortlaufend, was gerade auch auf dem ständigen Austausch mit ihrer weiteren natürlichen und kulturellen Umwelt beruht. Reichholf spricht insofern von „stabilen Ungleichgewichten“, in denen er ein zentrales Grundprinzip der „Ökologie der Zukunft“ sieht: Ökosysteme tendieren zu Veränderungen, nicht zur Konservierung von Gleichgewichtszuständen.

Aus kultureller Perspektive ist auch insbesondere die Technikentwicklung keineswegs auf die Stabilisierung von sozialen, ökonomischen und ökologischen Gleichgewichten angelegt. ${ }^{184}$ Mit technischen Innovationen wandelt sich nicht nur die Gesellschaft, sondern auch das Verhältnis von Kultur und Natur: Technische Interventionen verändern Ökosysteme. In diesem Verhältnis von Technik und Ökosystemen lassen sich neue Frontier-Perspektiven für das Anthropozän entfalten. ${ }^{185}$ Deshalb ist die anthropozäne Weltsicht auch keineswegs auf die oben beschriebene klaustrophobe Künstlichkeit der Welt festgelegt; in den Worten Peter Sloterdijks: „Auf dem Weg der bloßen Ausbeutung bleibt die Erde für alle Zeit die begrenzte Monade. Auf dem Weg der Koproduktion zwischen Natur und Technik könnte sie ein Hybridplanet werden, auf dem mehr möglich sein wird, als konservative Geologen glauben." 186 Aber auch dieser ko-produktive Weg von Natur und Technik wird konfliktgeladen sein: Welche Formen der Ko-Evolution von Natur und Technik sind die richtigen? Zur Beantwortung dieser Frage lassen sich in einem ersten Ansatz mit der Gewährleistung von „Resistenz“, „Resilienz“ und „Persistenz“ drei Konflikttypen konturieren, um anthropozäne Interessenkonflikte situationsgebunden zu bewältigen.

\section{Resistenz}

Die Gewährleistung der Resistenz eines Ökosystems greift als Konflikttypus immer dann, wenn durch eine kulturelle Intervention dessen vollkommene Funktionsaufhebung droht und insofern seine Existenz gefährdet wird. Vom Grundsatz her liegt dieser Konflikttypus auf der Traditionslinie der klassischen Gefahrenabwehr. Wenn also ein Ökosystem mit hinreichender Wahrscheinlichkeit zerstört zu werden droht, ist eine Abwägung vorzunehmen, die zu einem Eingriffsverbot führen kann, falls sich die ökosystemische Resistenz nicht durch technische Eingriffe garantieren lässt.

183 Vgl. hierzu und zum Folgenden J. H. Reichholf, Stabile Ungleichgewichte, Frankfurt 2008, S. 28 ff., 35 ff., 50 ff., 101 ff., 109 ff., 122 ff., 125 ff.

184 Vgl. H. Blumenberg, Geistesgeschichte der Technik, Frankfurt 2009, S. 26 f.

185 Vgl. Sloterdijk, Wie groß ist, "groß“? (Fn. 91), S. 108.

186 Sloterdijk, Wie groß ist „groß“? (Fn. 91), S. 109; vgl. ferner Fücks, Intelligent Wachsen, München 2013, S. 16, 42, 69, 117, 141 f., 150 ff., 163. 


\section{Resilienz}

Die Gewährleistung der Resilienz eines Ökosystems ist als Konflikttypus einschlägig, wenn eine Adaption von Kultur und Natur im Raum steht.

Der Begriff der „Resilienz“ umschreibt ein relativ neues Konzept. ${ }^{187}$ Resilienz hat sich insbesondere im Kontext von Vulnerabilitätskonzepten und Copingstrategien etabliert, um die Reaktion von kulturellen oder natürlichen Systemen zwischen Anpassung und Widerständigkeit im Hinblick auf kulturelle oder natürliche Umweltveränderungen zu beschreiben - mit anderen Worten: Das Konzept der „Resilienz“ funktioniert grundsätzlich sowohl bei der Veränderung der natürlichen Umwelt von kulturellen Systemen als auch - vice versa - bei der Veränderung der kulturellen Umwelt von Ökosystemen.

Dieses konzeptionelle Gegenstromprinzip begründet die besondere Bedeutung von Vulnerabilität, Coping und Resilienz im Anthropozän, das durch die Dynamik, Fragilität und Permeabilität des Verhältnisses von Kultur und Natur gekennzeichnet ist. Gerade deshalb ist es sinnvoll, das Anthropozän mit Donald Worster als ein „Age of Vulnerability“188 zu begreifen. Die Vulnerabilität eines kulturellen bzw. eines ökologischen Systems lässt sich dabei nach drei Kriterien bestimmen: ${ }^{189}$ erstens nach der Exposition, welche die Art, Häufigkeit und Intensität der Systembelastung durch natürliche oder kulturelle Einflüsse in den Blick nimmt; zweitens nach der Sensitivität, welche die Beeinflussung und Veränderung des exponierten Systems feststellt; und drittens nach der Anpassungsfähigkeit, die das Potenzial des betroffenen Systems reflektiert, sich den jeweiligen kulturellen und natürlichen Einflüssen anzupassen, etwa indem die Systemsensitivität reduziert oder die Systemauswirkungen kompensiert werden. Bei diesem dritten Vulnerabilitätskriterium setzt der Begriff der „Resilienz“ an, wenn er auf systemische Anpassungs- und Widerstandspotenziale fokussiert.

Im Hinblick auf die kulturelle Vulnerabilität durch Veränderungen der natürlichen Umwelt besteht die Gefahr, dass Copingstrategien in Form von kulturellen Eingriffen in die Natur die kulturelle Vulnerabilität nur noch weiter steigern. ${ }^{190}$ Das klassische historische Beispiel hierfür sind Flussbegradigungen, deren Reboundeffekte die kul-

187 Vgl. hierzu und zum Folgenden Ehlers, Anthropozän (Fn. 7), S. 16 f.; B. Allenby/J. Fink, Toward Inherently Secure and Resilient Societies, Science 309 (12.8.2005), 1034 ff.; C. Gusy, Resilient Societies, in: D. Heckmann/R. P. Schenke/G. Sydow (Hrsg.), Verfassungsstaatlichkeit im Wandel, Festschrift für Thomas Würtenberger, Berlin 2013, S. $995 \mathrm{ff}$.

188 D. Worster, The Age of Vulnerability (http://www.youtube.com/watch?v=kUZINBI3_SA [Abruf: 10.2.2014]); vgl. Clark/Crutzen/Schellnhuber, Science (Fn. 20), S. 18 ff.; Mauch, Mensch (Fn. 93), S. $49 \mathrm{ff}$.

189 Vgl. hierzu und zum Folgenden M. D. Mastrandrea/S. H. Schneider, Vorbereitungen für den Klimawandel, in: Crutzen/Davis/Mastrandrea/Schneider/Sloterdijk, Raumschiff (Fn. 88), S. 11 (48).

190 Vgl. Mauch, Mensch (Fn. 93), S. 60. 
turelle Verletzlichkeit immer wieder veranschaulicht haben ${ }^{191}$ und die faktisch, planerisch und politisch immer noch kaum beherrschbar sind. Dieser Befund hat sich durch die Verstärkung von Wetterextremen im Kontext des Klimawandels noch weiter verschärft. Zum Symbol kultureller Vulnerabilität ist die Zerstörung von New Orleans durch den Hurrikan Katrina im August und September 2005 geworden, der „das prekäre Zusammenspiel natürlicher Veränderung, kultureller Wahrnehmung, politischer Entscheidung, ökonomischen Kalküls und sozialer Benachteiligung “192 im Anthropozän offenbart hat.

Im Hinblick auf die ökologische Vulnerabilität durch Veränderungen der kulturellen Umwelt beschreibt Resilienz die Fähigkeit eines Ökosystems, mit Beeinträchtigungen und Störungen umzugehen. Kriterien dafür sind die Möglichkeiten eines Ökosystems, seine Basisfunktionen dynamisch zu entwickeln, sich an neue Umweltfaktoren anzupassen sowie Interventionen zu neutralisieren, zu kompensieren oder wiederum für die eigene Entwicklung zu nutzen. Mit dieser dynamischen Ausrichtung auf die Entwicklungsfähigkeit von Ökosystemen geht Resilienz über die Risikovorsorge hinaus. Resilienz mag das Vorsorgeprinzip durchaus einbeziehen, wird aber des Weiteren vor allem auch entwicklungsorientierte und experimentelle Formen der Konfliktlösung reflektieren.

Für die weitere konzeptionelle Konturierung des Konflikttypus der Resilienz lässt sich der Begriff der „Infrastruktur“ aufgreifen und ökologisch weiterentwickeln. In begriffsgeschichtlicher Perspektive thematisieren „Infrastrukturen“ das Verhältnis von technischer Entwicklung und sozialer Integration, das sich im Anthropozän in doppelter Hinsicht fortschreiben lässt: Zum einen kann die ökologische Dimension sehr viel stärker als bisher in die Regulierung sozialer und technischer Infrastrukturen eingebracht werden, sodass sich mit Christof Mauch programmatisch von einer „ökologischen Infrastruktur"193 sprechen lässt. Zum anderen kann man aber auch die soziale und technische Verletzlichkeit von Ökosystemen in den Metaphern der „Biotop-Infrastruktur“194 bzw. der „grüne[n] Infrastruktur der Natur“195 reflektieren, wenn es darum geht, deren Resilienz in der Wechselwirkung mit kulturellen Umwelteinflüssen zu bestimmen.

\section{Persistenz}

Die Gewährleistung der Persistenz eines Ökosystems kommt als Konflikttypus in Betracht, wenn dessen möglichst stabiles Fortbestehen langfristig garantiert werden soll. Hier kann die kulturelle oder - bereits spezifischer - die technische Intervention

191 Vgl. Mauch, Mensch (Fn. 93), S. $51 \mathrm{f}$.

192 Mauch, Mensch (Fn. 93), S. 54.

193 Vgl. Mauch, Mensch (Fn. 93), S. 68.

194 Markl, Natur (Fn. 6), S. 317.

195 Schwägerl, Menschenzeit (Fn. 2), S. 126; vgl. auch ders., ebd., S. 43. 
gerade notwendig sein, um die „natürliche“ Fortentwicklung eines Ökosystems zu verhindern und dessen Status in einer bestimmten Form zu konservieren, etwa um seltene Arten oder Landschaften zu erhalten. Die Bewertung solcher Konflikte um die ökologische Konservierung eines Ökosystems kann am Maßstab des Nachhaltigkeitsgrundsatzes erfolgen, der einen angemessenen Ausgleich zwischen sozialen, ökonomischen und ökologischen Interessen sucht. ${ }^{196}$

\section{Konfliktakteure}

Die rechtliche Repräsentation von Konflikten zwischen Kultur und Natur hängt nicht nur von der Ausdifferenzierung von Konflikttypen ab. Vielmehr kommt es auch ganz entscheidend auf die Anerkennung der beteiligten Konfliktakteure an. Doch die verfassungs- und europarechtlichen Umwelt- und Tierschutzbestimmungen vermitteln weder Menschen noch Tieren subjektive Rechte (Art. 20a GG; Art. 3 Abs. 3 Unterabs. 1 Satz 2 EUV; Art. 11, Art. 13, Art. 191 ff. AEUV; Art. 37 ChGrEU). ${ }^{197}$ Darüber hinaus greifen zwar im Hinblick auf schädliche Umwelteinwirkungen grundrechtliche Schutzpflichten für Leben und Gesundheit (Art. 2 Abs. 2 Satz 1 GG) sowie das Eigentum (Art. 14 Abs. 1 GG). ${ }^{198}$ Angesichts des weiten gesetzgeberischen Gestaltungsspielraums hat das Bundesverfassungsgericht allerdings bis heute noch in keinem umweltrechtlichen Fall die Verletzung grundrechtlicher Schutzpflichten beanstandet. ${ }^{199}$ Die Ökobilanz des Grundgesetzes und der europäischen Verträge fällt vor allem deshalb so mager aus, weil diese letztlich nur einen aktiven umweltpolitischen Akteur anerkennen: die öffentliche Gewalt, also insbesondere den Staat. Demgegenüber sollte aber eine anthropozäne Konfliktkultur gerade ihre menschlichen und nichtmenschlichen Akteurskonstellationen rechtlich phantasievoller entfalten, um ökologische Konflikte fairer und effektiver austragen zu können.

\section{Menschliche Akteure}

Im Hinblick auf die menschlichen Akteure gilt es, deren ökologischen Rechtsstatus für eine anthropozäne Konfliktkultur über die holozäne Verfassungslage hinaus weiterzuentwickeln. Im Zentrum der Überlegungen steht die Gewährleistung eines subjektiven Rechts aller menschlichen Akteure auf eine gesunde und saubere Umwelt. Die herrschende Meinung lehnt ein subjektives Recht auf Umweltschutz ab. ${ }^{200}$ Doch die Argumente gegen ein individuelles Grundrecht auf Schutz der eigenen Umwelt

196 Vgl. Schmidt/Kahl, Umweltrecht (Fn. 66), $\mathbb{1}$, Rdnr. 29 ff.; krit. Reichholf, Ungleichgewichte (Fn. 183), S. $118 \mathrm{f}$.

197 Vgl. für das Grundgesetz BVerfG, NVwZ 2010, S. 114 (115); für das Europarecht Jarass, ChGrEU, 2. Aufl., München 2013, Art. 37, Rdnr. 3.

198 Vgl. R. Sparwasser/R. Engel/A. Voßkuble, Umweltrecht, 5. Aufl., Heidelberg 2003, S. 51; Schmidt/ Kahl, Umweltrecht (Fn. 66), S. 79 f.

199 Vgl. A. Voßkuble, Umweltschutz und Grundgesetz, NVwZ 2013, S. 1 (7).

200 Vgl. BVerfG, NVwZ 2010, S. 114 (119f.); BVerwGE 54, 211, 220 f.; Steinberg, Der ökologische Verfassungsstaat, Frankfurt 1998, S. 421 ff.; M. Kloepfer, Zum Grundrecht auf Umweltschutz, Berlin/New York 1978, S. 14 ff. 
greifen nicht durch: Auf der Ebene der Landesverfassungen existieren durchaus subjektive Rechte auf Schutz vor Umweltgefährdungen: "Jeder hat" - nach Art.39 Abs. 2 BbgVerf - „das Recht auf Schutz seiner Unversehrtheit vor Verletzungen und unzumutbaren Gefährdungen, die aus Veränderungen der natürlichen Lebensgrundlagen entstehen.“ Darüber hinaus zeigt die Anerkennung eines „Grundrechts auf Naturgenuss“ (Art. 141 Abs. 3 Satz 1 BayVerf; Art. 10 Abs. 3 SächsVerf), dass in den Landesverfassungen sogar unmittelbar drittwirkende ökologische Grundrechtsgewährleistungen anerkannt sind. ${ }^{201}$ Ein solches Grundrecht auf Schutz der natürlichen Lebensgrundlagen ist darüber hinaus nicht auf einen allein abwehrrechtlichen Gehalt festgelegt, sondern kann sich auch in grundrechtlichen Teilhabe-, Verfahrens- und bei ökologischen Existenzbedrohungen - grundrechtlichen Leistungsansprüchen niederschlagen. Dies gilt selbst dann, wenn einzelne dieser Grundrechtsfunktionen durch den Gesetzgeber konkretisiert werden müssen, da dies bei „klassischen“ Grundrechten ebenfalls anerkannt wird. ${ }^{202}$ Deshalb ist eine grundrechtliche Ausdifferenzierung des ökologischen status libertatis, civitatis, activus und passivus der Bürgerinnen und Bürger im Anthropozän letztlich problemlos möglich.

\section{Nichtmenschliche Akteure}

Darüber hinaus kommt es für eine lebhafte anthropozäne Konfliktkultur darauf an, den Rechtsstatus nichtmenschlicher Akteure - also von Tieren, Naturgütern und Landschaften -zu entfalten. Als normativen Anknüpfungspunkt für die Konkretisierung eines subjektiven Rechtsstatus von Tieren, Naturgütern und Landschaften lässt sich grundsätzlich an die soeben genannten ökologischen Staats- und Unionszielbestimmungen anknüpfen. So kann der Gesetzgeber seinen etwa durch Art. 20a GG eröffneten rechtspolitischen Gestaltungssielraum dahingehend ausüben, dass er Tieren zu ihrem Schutz subjektive Rechte zuordnet. ${ }^{203}$ Dies gilt grundsätzlich auch für Naturgüter und Landschaften. Im Gegensatz zu diesen nur vom Gesetzgeber subjektivierbaren objektiv-rechtlichen Schutzklauseln ergibt sich aber überall dort unmittelbar aus der Verfassung ein subjektiver Rechtsstatus von Tieren, wo ausdrücklich deren „Achtung“ als „Lebewesen“ bzw. „Mitgeschöpf“ verankert ist (z.B. Art. 3b BWVerf; Art. 141 Abs. 1 Satz 2 BayVerf; Art. 6b NdsVerf; Art. 32 Satz 1 ThVerf). ${ }^{204}$ Denn mit dem Begriff der „Achtung“ geht in der Rechtsordnung regelmäßig die Anerkennung der subjektiven Rechtsstellung des Geachteten einher, die sich gerade nicht in der Gewährleistung objektiv-rechtlicher Schutzpflichten erschöpft. Soweit Verfassungen diesen Achtungsanspruch auf Pflanzen erstrecken, ver-

201 Vgl. BayVerfGH 28, 107 (125); 31, 198 (207); 47, 54 (57f.).

202 Vgl. C. Bumke/A. Voßkuble, Casebook Verfassungsrecht, Tübingen 2013, S. 68 ff.

203 Vgl. M. Kloepfer, in: W. Kahl/C. Waldhoff/C. Walter, Bonner Kommentar zum Grundgesetz, 162. Lfg., Heidelberg 2013, Art. 20a, Rdnr. 101 f.; a.A. R. Scholz, in: T. Maunz/G. Dürig, Grundgesetz, 69. Lfg., München 2013, Art. 20a, Rdnr. 70 ff.

204 Vgl. a.A. Scholz, in: Maunz/Dürig, Grundgesetz (Fn. 203), Art. 20a, Rdnr. 72 ff. 
fügen auch diese über einen subjektiven Rechtsstatus (Art. 39 Abs. 3 Satz 1 BbgVerf). Wenn auf diese Weise die Rechtssubjektivität von Tieren, Naturgütern und Landschaften verfassungsrechtlich begründet wird, ist der Gesetzgeber verpflichtet, diesen individuellen Rechtsstatus auch angemessen auszugestalten. Carolin Raspé hat in ihrer Studie über Die tierliche Person sehr anschaulich gezeigt, dass und wie eine (Neu)Positionierung der Tiere zwischen Subjekt- und Objektstatus im Rechtssystem möglich ist. ${ }^{205} \mathrm{Im}$ Mittelpunkt der Gewährleistung von Rechten für und an der tierlichen Person stehen deren physische Integritätsrechte sowie - unsicherer - die Rechte auf Existenzminimum und Bewegungsfreiheit, ${ }^{206}$ die auch in Form der Prozessstandschaft gerichtlich durchgesetzt werden können. ${ }^{207}$ Wie bereits im Fall der subjektiven Umweltrechte menschlicher Akteure bedeutet die Anerkennung von Tier- und Naturrechten nicht, dass sich diese in der Abwägung mit sozialen oder ökonomischen Interessen der Bürgerinnen und Bürger stets durchsetzen könnten bzw. müssten. Die Rechtssubjektivität und die Eigenrechte von Tieren, Naturgütern und Landschaften zeugt damit also keineswegs „von dem in jedem von uns mehr oder weniger ruhig schlummernden Wunsch, die verlorene Unschuld einer Welt wiederzufinden, in der die Pflanzen, die Tiere und die Objekte Mitbürger waren." ${ }^{208}$ Vielmehr geht es darum, auf der Grundlage der Rechtssubjektivität von Tieren, Naturgüter und Landschaften die Konflikte zwischen Kultur und Natur, die sich im Anthropozän immer weiter zuspitzen werden, angemessen austragen zu können. Aus diesem Grund werden Tiere, Pflanzen und Landschaften „nicht mehr an die Peripherie der Kollektive“209 gedrängt, sondern ihnen wird die Tür zum demokratischen und vor allem rechtsstaatlichen Diskurs geöffnet.

\section{Konzeptdimensionen}

Die Konfliktorientierung des Anthropozän-Konzepts schlägt sich ebenfalls in dessen vier Dimensionen - Zeit, Raum, Akteur und Wissenschaft - nieder. Insgesamt setzt das anthropozäne Konfliktkonzept auf eine adversative Ko-Evolution von Kultur und Natur. Immer neue Konfliktpotenziale und Konfliktkonstellationen begründen die evolutionäre Entwicklungsperspektive und dynamisieren auf diese Weise die zeitliche Dimension des Anthropozän. Diese konfliktbetonte Entwicklungsoffenheit spiegelt sich darüber hinaus im anthropozänen Raumbewusstsein: Zwar entfaltet sich die anthropozäne Konfliktkultur auf der einen Erde. Doch aufgrund technischen Innovationspotenzials, das als wissenschaftliche Dimension im konfligierenden Wechselspiel von Kultur und Natur angelegt ist, emanzipiert sich die anthropozäne

205 Vgl. Raspé, Die tierliche Person, Berlin 2013, S. 174 ff., 289 ff.

206 Vgl. Raspé, Person (Fn. 205), S. 313 ff.

207 Vgl. Raspé, Person (Fn. 205), S. $323 \mathrm{ff}$.

208 Descola, Jenseits von Natur und Kultur, Berlin 2011, S. 574.

209 Descola, Natur (Fn. 208), S. 571. 
Konfliktkultur von einer klaustrophoben Wahrnehmung unseres Planeten. Entwicklungsoffenheit prägt auch die Akteursdimension: Die anthropozäne Konfliktkultur erkennt neben menschlichen auch nichtmenschliche Konfliktakteure an und differenziert mit Resistenz, Resilienz und Persistenz drei Konflikttypen aus, um die ganze Komplexität der anthropozänen Welt zu repräsentieren.

\section{E. Fazit}

Die Erde ist in das Anthropozän eingetreten, in dem die Menschen zu einer bestimmenden geologischen Kraft geworden sind. Die damit einhergehende Verantwortung kommt nicht ohne ein Konzept menschlicher Selbstreflexion aus. Dieses Anthropozän-Konzept sollte nicht als Kontrakt im Sinn eines globalen Gesellschaftsvertrags für Nachhaltigkeit und auch nicht als postnatürliche Komposition gefasst werden. Es kann aber eine anthropozäne Konfliktkultur entfalten, die neben menschlichen auch nichtmenschliche Akteure anerkennt und die mit Resistenz, Resilienz und Persistenz über drei verschiedene Konflikttypen verfügt. Wenn auf diese Weise die konfliktgeladene Komplexität des Anthropozän konzipiert wird, bleibt dies allerdings nicht ohne Folgen für die politische Anthropologie. Die Provokation des anthropozänen „Menschenbilds“ liegt nicht in dem gern beschworenen ökologischen Calvinismus, der vor dem Hintergrund apokalyptischer Zukunftsszenarien eine ökologische „Umkehr“ und „Frugalität für alle“ ${ }^{210}$ fordert. ${ }^{211}$ Apokalyptische Worst-CaseSzenarien fördern vor allem Nach-mir-die-Sintflut-Einstellungen. ${ }^{212}$ Dennoch kommt das Anthropozän nicht ohne eine Entwicklung der politischen Anthropologie aus, die Peter Sloterdijk als eine Wende vom Homo Sapiens pauper zum Homo Sapiens luxus beschrieben hat: ${ }^{213}$ Die politische Moderne begreift den Menschen als „Mängelwesen“. ${ }^{214}$ Als das „noch nicht festgestellte Tier“ ${ }^{215}$ muss das menschliche Mängelwesen seine Defizite kulturtechnisch kompensieren. Diese Theorie anthropologischer Selbstviktimisierung hat normative wie kognitive Folgen: In normativer Hinsicht konsumiert der Homo Sapiens pauper nicht nur rein faktisch die natürlichen Ressourcen und zerstört die Umwelt. Vielmehr misst er sich selbst ein Recht auf Ressourcenverbrauch und Umweltzerstörung zu, um seine „Mangelfiktionen“ ${ }^{216} \mathrm{zu}$ kompensieren, quasi als normativ gewendete „Inkompetenzkompensationskompe-

210 Sloterdijk, Wie groß ist „groß“? (Fn. 91), S. 103.

211 Vgl. auch krit. Sloterdijk, Wie groß ist „groß“? (Fn. 91), S. 103, 105 f.; ders., Leben (Fn. 90), S. 709 ff.; Reich holf, Ungleichgewichte (Fn. 183), S. 120 f., 133 ff.

212 Vgl. Sloterdijk, Wie groß ist „groß“? (Fn. 91), S. 105.

213 Vgl. hierzu und zum Folgenden P. Sloterdijk, Sphären III, Frankfurt 2004, S. 699 ff.

214 A. Gehlen, Der Mensch, in: ders., Gesamtausgabe III/1, Frankfurt 1993, S. 16.

215 F. Nietzsche, Jenseits von Gut und Böse. Vorspiel einer Philosophie der Zukunft, in: ders., Werke (Fn. 83), S. 563 (623 [Nr. 62]).

216 Vgl. Sloterdijk, Übertreibungen (Fn. 90), S. 195, 235; vgl. ferner ders., ebd., S. 159. 
tenz “. ${ }^{217}$ In kognitiver Hinsicht immunisiert sich der Homo Sapiens pauper zugleich gegen die Wahrnehmung der faktischen Folgen seines eigenen Handelns: Er nimmt jede erdenkliche Komplexitätsreduzierung in Anspruch, weil er sich als Mängelwesen nur im Rahmen einer „Ökologie des Nichtwissens“ 218 orientieren zu können glaubt: Überall vermutet er „Komplexitätsfallen“. ${ }^{219}$ Doch für diesen Homo Sapiens pauper wäre das Anthropozän wohl keine sehr lange Erdperiode. Schon aus diesem Grund wird eine aufgeklärte Konfliktkultur des Anthropozän dieser abgeklärten politischen Anthropologie nicht folgen können. ${ }^{220}$ Denn die anthropozäne Konfliktkultur möchte die individuelle wie kollektive Wahrnehmung der sozialen, politischen, ökonomischen und ökologischen Komplexität unserer Welt gezielt steigern, gerade weil sie sich vor „Unterkomplexitätsfallen“ fürchtet. Eine anthropozäne Konfliktkultur kann die Menschen deshalb als Homo sapiens luxus begreifen, also als emotional wie kognitiv reiche Geschöpfe, die sich individuell und institutionell auf die ganze Komplexität ihrer anthropozänen Welt einlassen. Nur wenn wir uns immer wieder vergegenwärtigen, „daß es kein Menschenrecht auf Nicht-Überforderung gibt“, 221 werden wir keinen „plumpen“ und „einfallslosen Weg durch die Noosphä$\mathrm{re}^{\text {“222 }}$ gehen.

217 Vgl. zu diesem Begriff in einem freilich anderen Kontext O. Marquard, Inkompetenzkompensationskompetenz, in: ders., Der Abschied vom Prinzipiellen, Stuttgart 1981, S. 23.

218 N. Luhmann, Beobachtungen der Moderne, Opladen 1992, S. $149 \mathrm{ff.}$

219 M. Wehr, Die Komplexitätsfalle, FAZ, 17.11.2012, S. 40.

220 Vgl. Sloterdijk, Übertreibungen (Fn. 90), S. $421 \mathrm{f}$.

221 Sloterdijk, Leben (Fn. 90), S. 705; vgl. ferner ders., Übertreibungen (Fn. 90), S. 40.

222 D. Dath, Die Abschaffung der Arten, Frankfurt 2010, S. 27. 